\title{
A T-DMB navigation system for seamless positioning in both indoor and outdoor environments
}

\author{
Gong Bo Moon ${ }^{1}$, Se Bum Chun ${ }^{2}$, Moon Beom Hur ${ }^{2}$ and Gyu-In Jee ${ }^{1 *}$
}

\begin{abstract}
The conventional global positioning system (GPS) can often fail to provide position determination for a mobile user in indoor and urban environments. To cope with GPS failure in such environments, a new navigation system which utilizes a terrestrial digital multimedia broadcasting (T-DMB) signal to obtain the mobile user's position is presented. Since the T-DMB transmitters in Korea construct a single frequency network (SFN), which forces the transmitters to be synchronized, the mobile user can measure a time difference of arrival (TDOA) for all audible T-DMB transmitter pairs. The time difference between T-DMB transmitters is converted to a distance difference by multiplying the time difference by the speed of light. Using these measurements and a TDOA positioning method, the mobile user position can be estimated. An experiment with a T-DMB receiver and a data acquisition (DAQ) board is performed in Seoul to analyze the error characteristic of TDOA measurements. It is certified that the measurement error is bounded under $300 \mathrm{~m}$ and can be used to determine the mobile user's position with a small standard deviation.
\end{abstract}

Keywords: T-DMB positioning; TDOA; Indoor positioning; Seamless positioning; Phase reference symbol

\section{Introduction}

Recently, interest has been paid to the location-based service (LBS), which is used to provide more suitable information to a mobile user referring to his or her position. To achieve this goal, the mobile user position can be determined in both outdoor and indoor environments [1]. To date, the global positioning system (GPS) has provided positioning for the mobile user with sufficient accuracy in outdoor environments. GPS also covers a wide area with the aid of the many satellites surrounding the earth. In addition, small and lowcost GPS receiver chips enhance the user device mobility. However, the GPS signal on the ground is too weak to be received in an indoor environment. In addition, the high altitude of GPS satellites and skyscrapers in urban environments induce a multipath problem for the user receiver. The inherent characteristic of the GPS signal and the satellite constellation lead to an increase in errors for the mobile user's position. In addition, in the case when a number

\footnotetext{
* Correspondence: gijee@konkuk.ac.kr

${ }^{1}$ Department of Electrical Engineering, Konkuk University, Hwayang-dong, Gwangjin-gu, Seoul 143-701, South Korea

Full list of author information is available at the end of the article
}

of visible satellites decrease, the user receiver cannot determine the mobile user position.

To overcome these limitations of GPS in urban and indoor environments, many studies have been carried out with various radio sources, such as wireless local access network (Wi-Fi), cellular network, IEEE 802.15.4a (UWB), ultrasonic waves, Bluetooth, and DTV signals [2-16]. However, many of those positioning systems have limitations such as the requirement of pre-installation of the infrastructure for the positioning and/or data pre-processing for the region of interest. If the Wi-Fi signal is used for the mobile user position determination, building a received signal strength intensity (RSSI) map, which is called a fingerprint for an area of interest, is needed prior to the system operation. The positioning systems utilizing ultrasonic waves, bluetooth, and UWB need the installation of extra transmitters. Moreover, the service area for these positioning systems may be smaller than that of other positioning systems for the outdoor environment. The positioning method using the cellular communication channel confronts nonline-of-sight (NLOS) signal paths, because the base stations for the cellular network are located on the top of buildings in general. This may result in poor position estimate 
accuracy. In addition, to serve a seamless positioning in outdoor and indoor environments, the digital television (DTV) broadcasting signal is adopted. The positioning method utilizes a time of arrival (TOA) measurement between the DTV transmitter and the mobile user to determine the mobile user position [12]. Since the DTV transmitters are not synchronized with each other, the user receiver needs a monitoring station at a known position and an extra data channel to compensate the time offset of the DTV transmitters. A pre-installed monitoring station receives all audible DTV channel and calculates the time offset of the transmitters. This information can then be delivered to the user receiver via the extra communication channel.

To reduce these requirements, this paper presents a positioning system using a T-DMB signal for the seamless positioning. Since the T-DMB transmitters in Korea construct a single frequency network (SFN), which means that they are synchronized to each other, a time offset between the T-DMB transmitters are controlled to be zero using the GPS clock $[17,18]$. This means the T-DMB positioning method does not need the installation of a monitoring station and the extra communication channel. Also, the signal specifications force the T-DMB signal to include the position information of the T-DMB transmitters, which means that the mobile user does not need any prior information for the position determination $[19,20]$.

In this paper, a system structure for the T-DMB positioning method is presented. The proposed method utilizes the time difference of interval (TDOA) measurements between all audible T-DMB transmitter pairs and the TDOA positioning scheme. The experimental results and error analysis for TDOA measurements with the actual received T-DMB signals in Korea are presented. These results certify that the expected accuracy of the proposed positioning system is sufficient for use in the mobile user position determination of less than $200 \mathrm{~m}$ in most areas of Seoul in Korea. This performance may be worse than the GPS positioning in an outdoor environment, but the T-DMB positioning method maintains the performance even in indoor and urban environments, which provides the feasibility of the proposed system in the actual operation.

\section{T-DMB}

Terrestrial digital multimedia broadcasting (T-DMB) is derived from digital audio broadcasting (DAB), which is defined in the ETS 300401 standard. The DAB is developed by the European Standards Organization (ETSI) to serve the audio service via the digital broadcasting channel. Using error correction in the digital communication channel, the DAB can deliver the audio service with a better quality than that of analog broadcasting. Based on the $\mathrm{DAB}$, Korea developed the DMB, which includes not only an audio service but also a video service. The Korean Broadcast Act defines the DMB as a multiplexed broadcasting of video, audio, and data using multiple channels especially for reception during movement. In addition, the Comprehensive Plan for the Digital Multimedia Broadcasting states that the DMB is a digitalized multimedia broadcasting service, which is capable of transmitting an audio service of compact disc (CD) quality comparable to a video service with qualified reception at both rest and movement conditions. It can also be classified as terrestrial DMB (T-DMB) or satellite DMB (S-DMB) according to the broadcasting channel [21-24]. Nevertheless, the signal structure and broadcasting system follow the DAB standard.

\section{T-DMB signal structure}

In ETS 300 401, four DAB transmission modes are defined to allow the broadcasting system to be used for different network configurations and frequency bands as shown in Table 1. For example, the transmission mode $\mathrm{I}$ is designed for the single frequency network (SFN), such as the T-DMB in Korea, while the transmission mode IV is designed for the satellite DMB (SDMB) $[25,26]$.

As shown in Table 1, the T-DMB signal consists of transmission frames (Figure 1), which are a unit structure of the signal. Each transmission frame includes a synchronization channel (SC), a fast information channel (FIC), and a main service channel (MSC).

The SC delivers the null symbol and the phase reference symbol (PRS). The null symbol is inserted for the detection of transmission modes and the synchronization of the transmission frame. The null symbol is the first symbol for every transmission frame. For the null symbol duration, the actual transmitted signal is equal to zero. This characteristic helps the T-DMB receiver to measure the time duration of the null symbol for determining the transmission mode from I to IV. In addition, the phase reference symbol (PRS), which is the first orthogonal frequency division multiplex (OFDM) symbol followed the null symbol to provide the reference phase information for the demodulation of the remaining OFDM symbols after the PRS. For this purpose, the DAB standard, ETS

\section{Table 1 DAB transmission mode}

\begin{tabular}{lccc}
\hline $\begin{array}{l}\text { Transmission } \\
\text { mode }\end{array}$ & $\begin{array}{c}\text { Duration of } \\
\text { transmission } \\
\text { frame }\end{array}$ & $\begin{array}{c}\text { Number of } \mathrm{FIB}_{\mathbf{s}} \\
\text { per transmission } \\
\text { frame }\end{array}$ & $\begin{array}{c}\text { Number of } \mathrm{CIF}_{\mathbf{s}} \\
\text { per transmission } \\
\text { frame }\end{array}$ \\
\hline I & $96 \mathrm{~ms}$ & 12 & 4 \\
III & $24 \mathrm{~ms}$ & 3 & 1 \\
IV & $24 \mathrm{~ms}$ & 4 & 1 \\
\hline
\end{tabular}




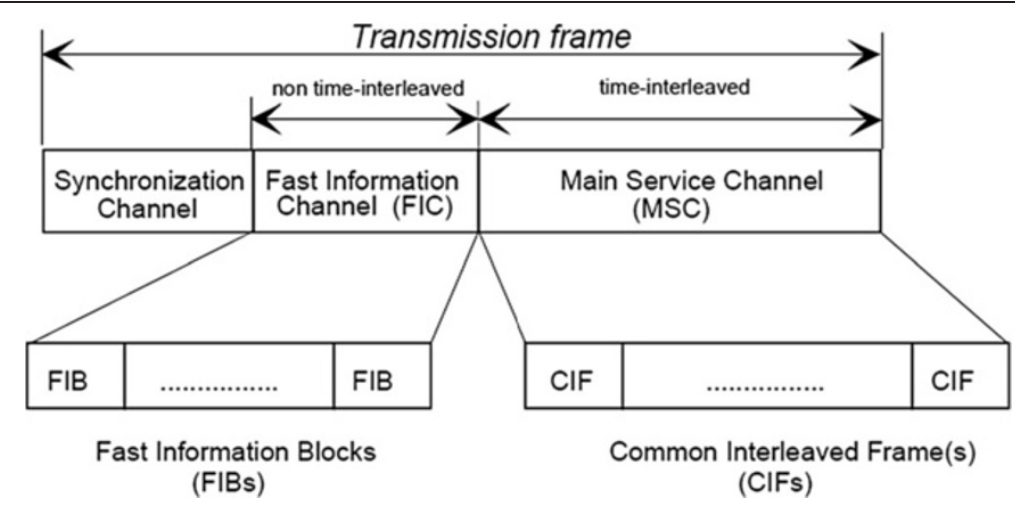

Figure 1 T-DMB transmission frame.

300401 , specifies the characteristics of the null symbol and the PRS. The same specification applies to the TDMB signal. To examine the $\mathrm{SC}$, parameters and variables which are related to the T-DMB signal generation process are defined as follows:

$L$ is the number of OFDM symbols per transmission frame (the null symbol is excluded).

$K$ is the number of transmitted carriers.

$T_{\mathrm{F}}$ is the transmission frame duration.

$T_{\mathrm{NULL}}$ is the null symbol duration.

$T_{\mathrm{S}}$ is the duration of OFDM symbols of indices for $l=$ $1,2,3, \ldots, L$.

$T_{\mathrm{U}}$ is the inverse of the carrier spacing.

$\Delta$ is the duration of the time interval called the guard interval.

$Z_{m, l, k}$ is the complex D-QPSK symbol associated with the $k$-th carrier of the $l$-th OFDM symbol during the $m$-th transmission frame.

The values of the parameters mentioned above, excluding $Z_{m, l k}$, are specified in Table 2 for transmission modes I, II, III, and IV.

With the above parameters and calculated values, the main signal $s(t)$ for the T-DMB (transmission mode I of the $\mathrm{DAB})$ is defined in Equation 1 [19].

$s(t)=\operatorname{Re}\left[e^{2 j \pi f_{c} t} \sum_{m=-\infty}^{+\infty} \sum_{l=0}^{L} \sum_{k=-\frac{K}{2}}^{\frac{K}{2}} z_{m, l, k} \times g_{k, l}\left(t-m \times T_{F}-T_{\mathrm{NULL}}-(l-1) \times T_{S}\right)\right]$

Here,

$$
\begin{aligned}
g_{k, l}(t) & =\left\{\begin{array}{lc}
\frac{2 j \pi k(t-\Delta)^{0}}{T_{U}} \cdot \operatorname{Rect}\left(\frac{t}{T_{S}}\right) & \text { for } l=0 \\
T_{\mathrm{S}} & =T_{\mathrm{U}}+\Delta,
\end{array}\right.
\end{aligned}
$$

$$
\operatorname{Rect}(t)=\left\{\begin{array}{cc}
1 & \text { for } 0 \leq t<1 \\
0 & \text { otherwise }
\end{array} .\right.
$$

In Equation 1, the subscripts of $m, l$, and $k$ represent the sequential number of the current frame from the beginning of transmission, the index of the current OFDM symbol, and the index of the associating carrier, respectively. If we apply the actual argument $t-m \times T_{F}-T_{\mathrm{NULL}}-$ $(l-1) \times T_{\mathrm{S}}$ for the function $g_{k, l}(t)$, then $-m \times T_{\mathrm{F}}$ maintains the beginning of the current frame as $t=0$. Next, $-T_{\mathrm{NULL}}$ eliminates the effects of the null symbol in the time axis, so that the beginning of each PRS can be interpreted as the time origin for the signal generating process in the T-DMB transmitter. In addition, for the PRS, $l=1$ can be applied to remove the last term $-(l-1) \times T_{\mathrm{S}}$. Finally, the output of $g_{k, l}(\cdot)$ for the PRS in the time interval of $t=\left[0, T_{\mathrm{S}}\right]$ at any time can be verified, which means that the phase reference symbol is time invariant.

If we notice that the complex D-QPSK symbol $z_{m, l, k}$ for the phase reference symbols has fixed values, the exponential terms in $g_{k, l}(t)$ can be interpreted as the Fourier transform coefficients. This means that the main signal for the PRS is the Fourier transform results of the corresponding D-QPSK symbol $z_{m, l, k}$. The carrier frequency spacing for the Fourier transform is $1 \mathrm{KHz}$, as specified in ETS 300401.

Moreover, for the complex D-QPSK symbol $z_{m, l, k}$ corresponding to the PRS, $m$ can be ignored, and $l=1$ can be applied. Therefore, the complex D-QPSK symbol $z_{m, l, k}$ for the PRS can be rewritten as $z_{1, k}$. The $z_{m, l, k}$ can then be calculated using Equation 2, Table 3, and Table 4.

$$
z_{1, k}=\left\{\begin{array}{cc}
e^{j \phi_{k}} & \text { for }-\frac{K}{2} \leq k<0 \text { and } 0<k \leq \frac{K}{2} \\
0 & \text { for } k=0
\end{array}\right.
$$

Here, $\phi_{k}=\frac{\pi}{2}\left(h_{i, k-k^{\prime}}+n\right)$, and $i, k^{\prime}$, and $n$ are specified in Table 3 for the carrier number $k$, and $h_{i, k}-k^{\prime}$ is specified in Table 4. 
Table 2 Definition of the parameters for transmission modes

\begin{tabular}{lcccc}
\hline Parameter & Transmission mode I & Transmission mode II & Transmission mode III & Transmission mode IV \\
\hline$L$ & 76 & 76 & 153 & 76 \\
$K$ & 1,536 & 384 & 192 & 768 \\
$T_{\mathrm{F}}$ & $196,608 \mathrm{~T}$ & $49,152 \mathrm{~T}$ & $49,152 \mathrm{~T}$ & $98,304 \mathrm{~T}$ \\
& $96 \mathrm{MS}$ & $24 \mathrm{MS}$ & $24 \mathrm{MS}$ & $48 \mathrm{MS}$ \\
$T_{\text {NULL }}$ & $2656 \mathrm{~T}$ & $664 \mathrm{~T}$ & $345 \mathrm{~T}$ & $1,328 \mathrm{~T}$ \\
& $1297 \mathrm{~ms}$ & $-324 \mu \mathrm{s}$ & $-168 \mu \mathrm{s}$ & $-648 \mu \mathrm{s}$ \\
$T_{\mathrm{S}}$ & $2552 \mathrm{~T}$ & $638 \mathrm{~T}$ & $319 \mathrm{~T}$ & $1,276 \mathrm{~T}$ \\
& Approximately $1,246 \mathrm{~ms}$ & Approximately $312 \mu \mathrm{s}$ & Approximately $156 \mu \mathrm{s}$ & Approximately $623 \mu \mathrm{s}$ \\
$T_{U}$ & $2,048 \mathrm{~T}$ & $512 \mathrm{~T}$ & $256 \mathrm{~T}$ & $1,024 \mathrm{~T}$ \\
& $1 \mathrm{~ms}$ & $250 \mu \mathrm{s}$ & $125 \mu \mathrm{s}$ & $500 \mu \mathrm{s}$ \\
$\Delta$ & $504 \mathrm{~T}$ & $126 \mathrm{~T}$ & $63 \mathrm{~T}$ & $252 \mathrm{~T}$ \\
& Approximately $246 \mu \mathrm{s}$ & Approximately $62 \mu \mathrm{s}$ & Approximately $31 \mu \mathrm{s}$ & Approximately $123 \mu \mathrm{s}$ \\
\hline
\end{tabular}

Using Equation 2, the D-QPSK symbol for the PRS can be generated as shown in Figure 2 .

The result shows that $z_{1, k}$ takes one of the values in $\{1$, $j,-1,-j\}$, which constructs the constellation on the complex plane. Using the above results, an ideal transmission signal for the $\mathrm{SC}$ can be constructed. For the time duration of $\left[0, T_{\mathrm{NULL}}\right]$, which corresponds to the null symbol, the main signal of transmission remains as zero because the output of $g_{k, l}(\cdot)$ is zero for that time interval. This signal characteristic can be used to detect the $\mathrm{SC}$ in the T-DMB receiver as mentioned earlier. Also, the following signal for the PRS can be generated by applying the results of Equation 2 to Equation 1 .

$$
\begin{aligned}
& s(t)=\operatorname{Re}\left[e^{2 j \pi f_{c} t} \sum_{k=-\frac{K}{2}}^{\frac{K}{2}} z_{1, k} \times g_{k, l}\left(t-T_{\mathrm{NULL}}\right)\right] \\
& =\operatorname{Re}\left[e^{2 j \pi f_{c} t} \sum_{k=-\frac{K}{2}}^{\frac{K}{2}} z_{1, k} \times e^{\frac{2 j \pi k\left(t-T_{\mathrm{NULL}}-\Delta\right)}{T_{\mathrm{U}}}} \times \operatorname{Rect}\left(\frac{t-T_{\mathrm{NULL}}}{T_{\mathrm{S}}}\right)\right] \\
& =\operatorname{Re}\left[e^{2 j \pi f_{c} t} \sum_{k=-\frac{K}{2}}^{\frac{K}{2}} z_{1, k} \times e^{\frac{2 j \pi k\left(t-T_{\mathrm{NULL}}-\Delta\right)}{T_{\mathrm{U}}}}\right]
\end{aligned}
$$

Since the relative time interval for the PRS in the transmission frame is $\left[T_{\mathrm{NULL}}, T_{\mathrm{NULL}}+T_{\mathrm{S}}\right]$, $\operatorname{Rect}(\cdot)$ in Equation 3 holds as 1 for that time interval. Using Equation 3, the ideal main signal for the SC corresponding to the 12A channel (Munhwa Broadcasting Cooperation), which has a center frequency of $205.28 \mathrm{MHz}$, is generated and illustrated in Figure 3.

\section{T-DMB signal processing}

\section{Frame synchronization}

As mentioned above, the actual transmission signal holds as zero during the null symbol duration in the SC. This means that a sum of the signal energy for the null symbol is smaller than that of any other symbols. This characteristic enables the T-DMB receiver to find the beginning point of the transmission frame [27-29]. This would serve as a reference point for finding a specific part of the transmitted signal corresponding to the PRS at the T-DMB receiver. This is called coarse frame synchronization, and the process is as follows: Two sequential sample windows are set on a received T-DMB intermediate frequency (IF) signal to calculate the energy sum for corresponding signals as shown in Figure 4.

The window length $W$ should be smaller than the length of an OFDM symbol to maximize the difference of energy sum between the null symbol and any other symbols. The energy sums in two sample windows are represented as $W_{1}$ and $W_{2}$, respectively. Then, the ratio of energy sum for two sample windows is written as $P_{\delta}$ in Equation 4.

$$
P_{\delta}=\frac{W_{1}}{W_{2}}=\frac{\sum_{t=-W}^{-1}|s(t+\delta)|^{2}}{\sum_{t=0}^{W-1}|s(t+\delta)|^{2}}
$$

Here, $\delta$ is a sample index, which refers to a position of the first sample in the second sample window, $W_{2}$.

As two sample windows move to the right on the sampled T-DMB signal by a moving step, the energy ratio $P_{\delta}$ and the sample index $\delta$ are stored. When the sample index $\delta$ is placed at the first sample of the null symbol, the energy ratio $P_{\delta}$ would have a maximum value. Usually, to synchronize the frame more rapidly, the moving 
Table 3 Predefined indices $\boldsymbol{i}, \boldsymbol{k}^{\prime}$, and $\boldsymbol{n}$ by the carrier index $k$ (for transmission mode I)

\begin{tabular}{|c|c|c|c|c|}
\hline \multicolumn{2}{|c|}{$k$} & \multirow[t]{2}{*}{$k^{\prime}$} & \multirow[t]{2}{*}{$i$} & \multirow[t]{2}{*}{$n$} \\
\hline $\min$ & $\max$ & & & \\
\hline-768 & $\begin{array}{l}-737 \\
\end{array}$ & -768 & 0 & $\overline{1}$ \\
\hline-768 & -705 & -736 & 1 & 2 \\
\hline-704 & -673 & -704 & 2 & 0 \\
\hline-672 & -641 & -672 & 3 & 1 \\
\hline-640 & -609 & -640 & 0 & 3 \\
\hline-608 & -577 & -608 & 1 & 2 \\
\hline-576 & -545 & -576 & 2 & 2 \\
\hline-544 & 513 & -544 & 3 & 3 \\
\hline-512 & -481 & -512 & 0 & 2 \\
\hline-480 & -449 & 480 & 1 & 1 \\
\hline-448 & -417 & -448 & 2 & 2 \\
\hline-416 & -385 & -416 & 3 & 3 \\
\hline-384 & -353 & -384 & 0 & 1 \\
\hline-352 & -321 & -352 & 1 & 2 \\
\hline-320 & -286 & -320 & 2 & 3 \\
\hline-288 & -257 & -288 & 3 & 3 \\
\hline-256 & -225 & -256 & 0 & 2 \\
\hline-224 & -193 & -224 & 1 & 2 \\
\hline-192 & -161 & -192 & 2 & 2 \\
\hline-160 & -129 & -160 & 3 & 1 \\
\hline-128 & -97 & -128 & 0 & 1 \\
\hline-96 & -65 & -96 & 1 & 3 \\
\hline-64 & -33 & -64 & 2 & 1 \\
\hline-32 & -1 & -32 & 3 & 2 \\
\hline 1 & 32 & 1 & 0 & 3 \\
\hline 33 & 64 & 33 & 3 & 1 \\
\hline 65 & 96 & 65 & 2 & 1 \\
\hline 97 & 128 & 97 & 1 & 1 \\
\hline 129 & 160 & 129 & 0 & 2 \\
\hline 161 & 192 & 161 & 3 & 2 \\
\hline 193 & 224 & 193 & 2 & 1 \\
\hline 225 & 256 & 225 & 1 & 0 \\
\hline 257 & 288 & 257 & 0 & 2 \\
\hline 289 & 320 & 289 & 3 & 2 \\
\hline 321 & 352 & 321 & 2 & 3 \\
\hline 353 & 384 & 353 & 1 & 3 \\
\hline 385 & 416 & 385 & 0 & 0 \\
\hline 417 & 448 & 417 & 3 & 2 \\
\hline 449 & 480 & 449 & 2 & 1 \\
\hline 481 & 512 & 481 & 1 & 3 \\
\hline 513 & 544 & 513 & 0 & 3 \\
\hline 545 & 576 & 545 & 3 & 3 \\
\hline
\end{tabular}

Table 3 Predefined indices $\boldsymbol{i}, \boldsymbol{k}^{\prime}$, and $\boldsymbol{n}$ by the carrier index $k$ (for transmission mode I) (Continued)

\begin{tabular}{lllll}
\hline 577 & 608 & 577 & 2 & 3 \\
609 & 640 & 609 & 1 & 3 \\
641 & 672 & 641 & 0 & 3 \\
673 & 704 & 673 & 6 & 0 \\
705 & 736 & 705 & 2 & 1 \\
737 & 768 & 737 & 1 & 1 \\
\hline
\end{tabular}

step for sample windows is 10 or 20 samples, which is the reason why it is called coarse frame synchronization. The above process can be formulated as follows in Equation 5.

$$
\begin{aligned}
\hat{t}_{\text {coarse sync }} & =\arg \max _{\delta}\left(P_{\delta}\right) \times T_{\text {sample }} \\
& =\arg \max _{\delta} \frac{\sum_{t=-W}^{-1}|s(t+\delta)|^{2}}{\sum_{t=0}^{W-1}|s(t+\delta)|^{2}} \times T_{\text {sample }}
\end{aligned}
$$

Here, $\delta$ is the sample index, which corresponds to the first sample in the second sample window, and $T_{\text {sample }}$ refers to the sample time in a T-DMB receiver.

The coarse frame synchronization method is applied to the sampled data (Figure 5), which is acquired from the T-DMB receiver, to certify the introduced method. Since the received signal at radio frequency (RF) stage is modulated by the high frequency of the broadcasting channel's center frequency in the range of 170 to $210 \mathrm{MHz}$, an IF signal is used. The IF signals are the output of the RF tuner in the T-DMB receiver.

In general, the RF signal is processed in the RF tuner device to eliminate the center frequency component of the transmission channel by down-converting to a baseband frequency. This helps the digital signal processor (DSP) in the baseband to handle the receiving signal at a lower-frequency range. Using this technique reduces the complexity of the receiver, which also reduces costs. In addition, the RF tuner with the IF stage output can be adapted to other basebands with different sampling rates.

Figure 5 shows that $P_{\delta}$, which is the energy ratio of two sequential sample windows, is at a maximum at every $96 \mathrm{~ms}$, which is the duration of every single transmission frame in the T-DMB. Corresponding sample points indicate the beginning points of three sequential transmission frames, since the null symbol leads the transmission frame. Figure 6 shows the actual IF signal for the null symbol, which is extracted using the coarse frame synchronization method. To show that the signal strength of the null symbol is much smaller than any 
Table 4 Time-frequency-phase parameter $h$

\begin{tabular}{|c|c|c|c|c|c|c|c|c|c|c|c|c|c|c|c|c|}
\hline$j$ & 0 & 1 & 2 & 3 & 4 & 5 & 6 & 7 & 8 & 9 & 10 & 11 & 12 & 13 & 14 & 15 \\
\hline$h_{0, j}$ & 0 & 2 & 0 & 0 & 0 & 0 & 1 & 1 & 2 & 0 & 0 & 0 & 2 & 2 & 1 & 1 \\
\hline$h_{1, j}$ & 0 & 3 & 2 & 3 & 0 & 1 & 3 & 0 & 2 & 1 & 2 & 3 & 2 & 3 & 3 & 0 \\
\hline$h_{2, j}$ & 0 & 0 & 0 & 2 & 0 & 2 & 1 & 3 & 2 & 2 & 0 & 2 & 2 & 0 & 1 & 3 \\
\hline$h_{3, j} j$ & 0 & 1 & 2 & 1 & 0 & 3 & 3 & 2 & 2 & 3 & 2 & 1 & 2 & 1 & 3 & 2 \\
\hline$j$ & 16 & 17 & 18 & 19 & 20 & 21 & 22 & 23 & 24 & 25 & 26 & 27 & 28 & 29 & 30 & 31 \\
\hline$h_{0, j}$ & 0 & 2 & 0 & 0 & 0 & 0 & 1 & 1 & 2 & 0 & 0 & 0 & 2 & 2 & 1 & 1 \\
\hline$h_{1, j}$ & 0 & 3 & 2 & 3 & 0 & 1 & 3 & 0 & 2 & 1 & 2 & 3 & 2 & 3 & 3 & 0 \\
\hline$h_{2, j}$ & 0 & 0 & 0 & 2 & 0 & 2 & 1 & 3 & 2 & 2 & 0 & 2 & 2 & 0 & 1 & 3 \\
\hline$h_{3, j}$ & 0 & 1 & 2 & 1 & 0 & 3 & 3 & 2 & 2 & 3 & 2 & 1 & 2 & 1 & 3 & 2 \\
\hline
\end{tabular}

other symbols, the closing part of the last symbol in the previous frame and the beginning part of the PRS are included in the figure. In Figure 6, the two vertical solid lines represent the beginning and ending of the null symbol, while the dotted line is the energy ratio (shown in Figure 5). In addition, the null symbol is multiplied by 15 to present the signal and ratio in a single view.

Due to the signal structure of the OFDM symbol, the fine frame synchronization method can be applied after the coarse frame synchronization to find a more exact beginning point of the transmission frame. This is because the first leading part of an OFDM symbol is a replica of the last part of the OFDM symbol and is inserted intentionally to serve as a guard interval to avoid the ISI, which occurs when a delayed signal or a multipath occurs in a transmission channel. The replica signal is called a cyclic prefix (CP) because the insertion of that signal resembles the cyclic shift process to the right direction. In transmission mode I, the time duration of a CP is specified as $246 \mu \mathrm{s}$, which is a 504 multiple of a basic period $T$. Since the T-DMB transmitters in Korea form a SFN to utilize the spectrum resources effectively, the maximum distance between the transmitters is limited by the length of the CP. For the T$\mathrm{DMB}$, the time duration of the CP, which is $246 \mu \mathrm{s}$, corresponds to the distance of $73.8 \mathrm{~km}$.

The result of the coarse frame synchronization provides an initial estimation of the beginning point for the transmission frame. Using this reference and the DAB standards, the beginning point of the PRS can be calculated. This means that the signal for the PRS can be extracted from the received T-DMB signal as shown in Figure 7, and the two dotted lines represent the beginning and closing of the IF signal corresponding to the PRS.

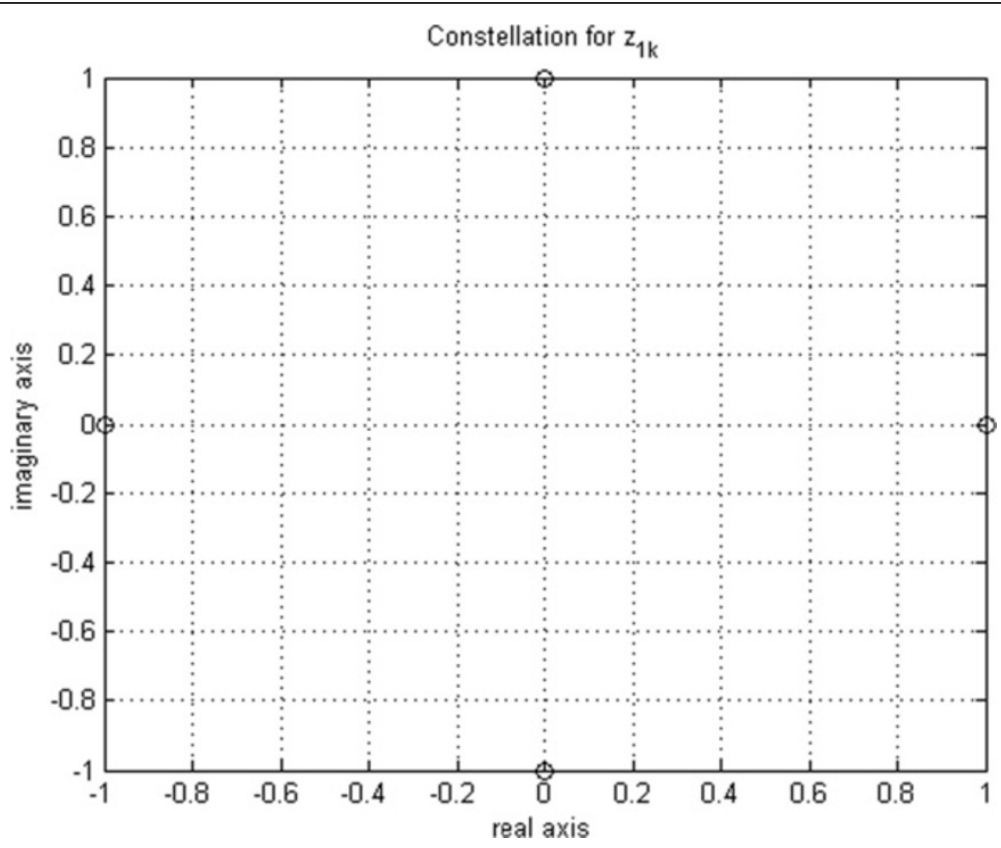

Figure $2 Z_{1, k}$, the D-QPSK symbol for the PRS. 


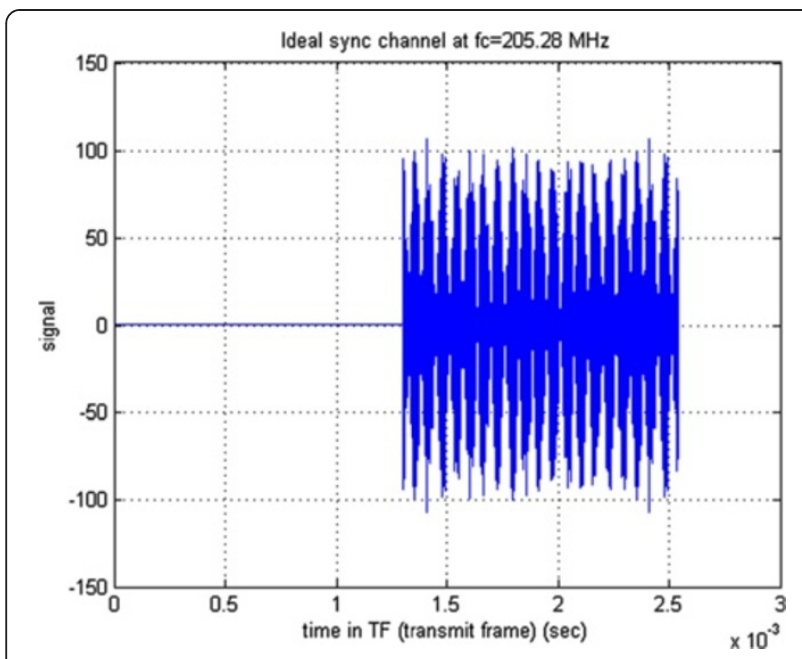

Figure 3 Ideal synchronization channel.

If it is assumed that the sampled IF signal is $s(t)$, the estimated IF signal for the PRS is $P_{\mathrm{IF}}(t)$, and the beginning point of the PRS in the sampled IF signal is $\hat{t}_{0, \text { PRS }}$; the estimated PRS can then be written as follows:

$$
P_{\mathrm{IF}}(t)=s(t) \quad \text { for } \hat{t}_{0, \mathrm{PRS}} \leq t \leq \hat{t}_{0, \mathrm{PRS}}+\Delta+T_{\mathrm{U}}
$$

Here, $T_{\mathrm{U}}$ is the time duration of an OFDM symbol in the T-DMB (excluding the $\mathrm{CP}$ ), and $\Delta$ is the time duration of the CP in the OFDM symbol.

If there is no estimation error, $\delta t_{0, \mathrm{PRS}}$, for the beginning point of the PRS, the sampled IF signal in the interval of $\left[\hat{t}_{0, \mathrm{PRS}}, \hat{t}_{0, \mathrm{PRS}}+\Delta\right]$ corresponding to the CP of the PRS is exactly the same as the original signal in the interval of $\left[\hat{t}_{0, \mathrm{PRS}}+T_{\mathrm{U}}, \hat{t}_{0, \mathrm{PRS}}+\Delta+T_{\mathrm{U}}\right]$ as specified in the DAB standards.

$$
\left.P_{\mathrm{IF}}(t)\right|_{\left.\hat{t}_{0, \mathrm{PRS}}, \hat{t}_{0, \mathrm{PPS}}+\Delta\right]}=\left.P_{\mathrm{IF}}(t)\right|_{\left[\hat{t}_{0, \mathrm{PRS}}+T_{\mathrm{U}}, \hat{t}_{0, \mathrm{PPS}}+\Delta+T_{\mathrm{U}}\right]} \text { if } \delta t_{0, \mathrm{PRS}}=0
$$

Since the $\mathrm{CP}$ is designed to be the same as the signal of the last part of the OFDM symbol to avoid the ISI, the cross-correlation of two signals has a maximum value when no estimation error $\delta t_{0, \mathrm{PRS}}$ occurs. This characteristic can be applied to perform the fine frame synchronization as follows in Equation 8.

$$
\delta \hat{t}_{0, \mathrm{PRS}}=\arg \max _{\delta} C_{\delta}(\tau=0)
$$

In here, $C_{\delta}(\cdot)$ is the cross-correlation of two signal.

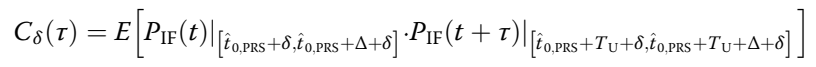

Equation 8 is applied to the actual sampled data to certify the fine frame synchronization. Figure 8 shows the result of the fine frame synchronization method, where the $x$ axis refers to the estimated frame synchronization error, and the $y$ axis refers to the magnitude for the cross-correlation of estimated signals with the time delay $\tau$, which is zero.

The estimated PRS after the correction of the synchronization error using the fine frame synchronization is illustrated in Figure 9. Compared to the primary estimation using the coarse frame synchronization in Figure 9, it can be shown that the fine frame synchronization found a more exact beginning point of the PRS.

\section{TDOA measurement using PRS}

As mentioned, the PRS can be found after the frame synchronization process. Then, the PRS is used to measure a time difference of arrival (TDOA) from multiple T-DMB transmitters because it has an impulse characteristic in an autocorrelation. For example, the autocorrelation result of the ideal SC in time domain is shown in Figure 10.

As expected, the result shows the impulse characteristic at the origin of the time axis. The occurrences of two small peaks on both sides of the main peak are caused by the cyclic prefix $(\mathrm{CP})$ in the PRS. Each OFDM symbol begins with the $\mathrm{CP}$, which is an intentionally inserted replica of the last part of the current symbol, to prevent the inter symbol interference (ISI) by the transmission signal and its delayed signal. In the T-DMB standard, a time interval between the $\mathrm{CP}$ and the original signal is $1 \mathrm{~ms}$ as shown in Figure 10. This is the reason why the side peaks are at $1 \mathrm{~ms}$ apart from the origin of the time axis.

However, the autocorrelation using the actual received PRS shows a different aspect. Figure 11 illustrates the autocorrelation result of the sampled PRS at the IF stage in the actual received T-DMB signal.

In Figure 11, the autocorrelation result includes extra side peaks around the center peak, which are not observed in the case of ideal PRS. These are obtained by

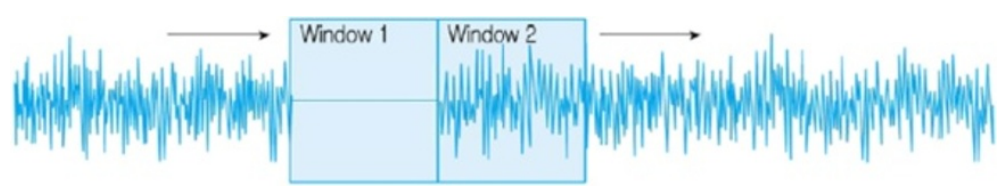

Figure 4 Sample windows for the coarse frame synchronization. 

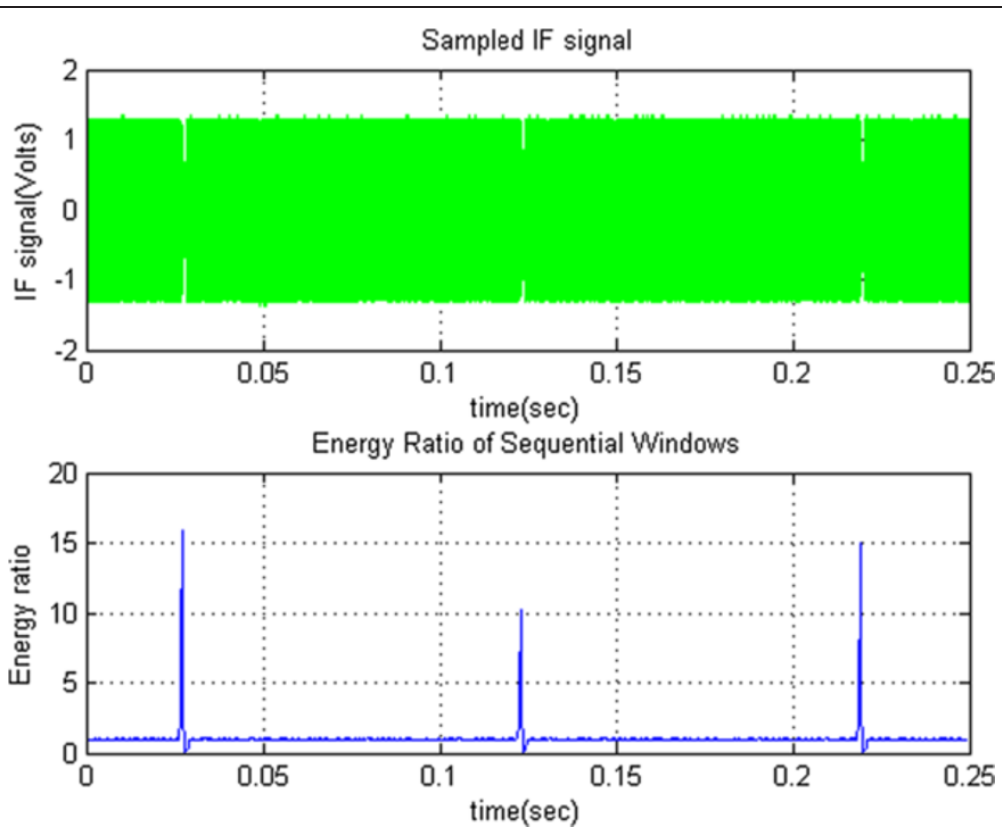

Figure 5 Coarse frame synchronization using the actual T-DMB signal (IF).

the cross-correlation between the first arrived signal from a T-DMB transmitter and the following signal from another T-DMB transmitter. Since the same T-DMB channel uses the same center frequency, a receiver should see transmission signals from multiple transmitters as a sum of the original signals, and it is a scaleddown replica with a time delay. If there are two transmitters with different distances to the mobile user, the transmission signal from the closest transmitter corresponds to the original signal, and the other transmission signal can be considered as the replica. Due to the longer distance, the following signal should be scaled down

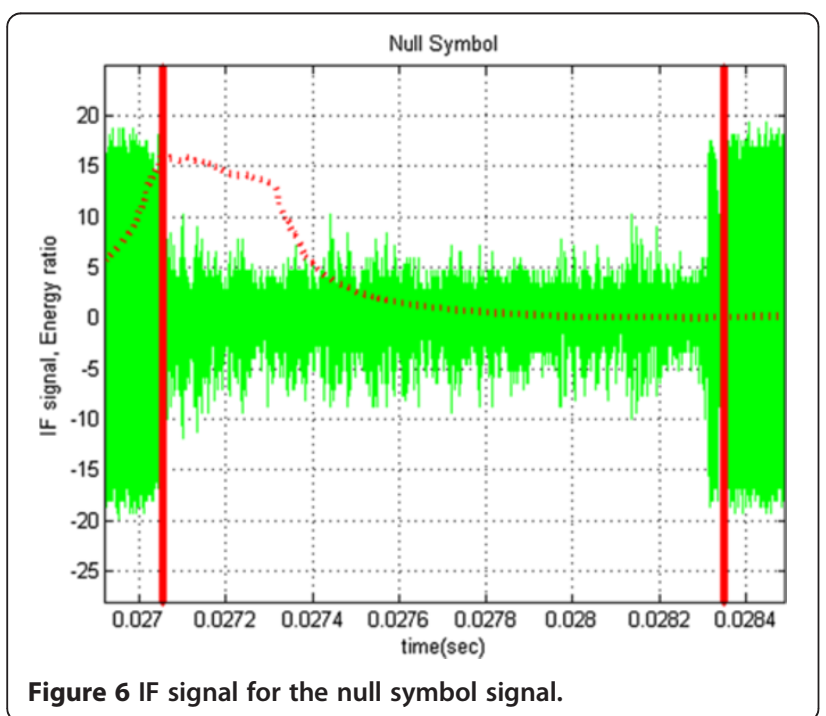

and time delayed. If we consider the case of an ideal reception, the extracted PRS signals from those two received signals at the IF stage can be written as $P_{\text {first_ }}$ arrived $(t)$ and $P_{\text {second_arrived }}(t)$. Then, Equation 9 holds for these signals.

$$
P_{\text {second_arrived }}(t)=\alpha_{\text {relative }} \times P_{\text {first_arrived }}\left(t-t_{\mathrm{d}}\right)
$$

Here, $\alpha_{\text {relative }}$ is a scale factor and $t_{\mathrm{d}}$ is a delay time for the second arrived signal.

Using Equation 9, an ideal received signal, $P_{\text {receiver }}(t)$, at the IF stage can be written as Equation 10.

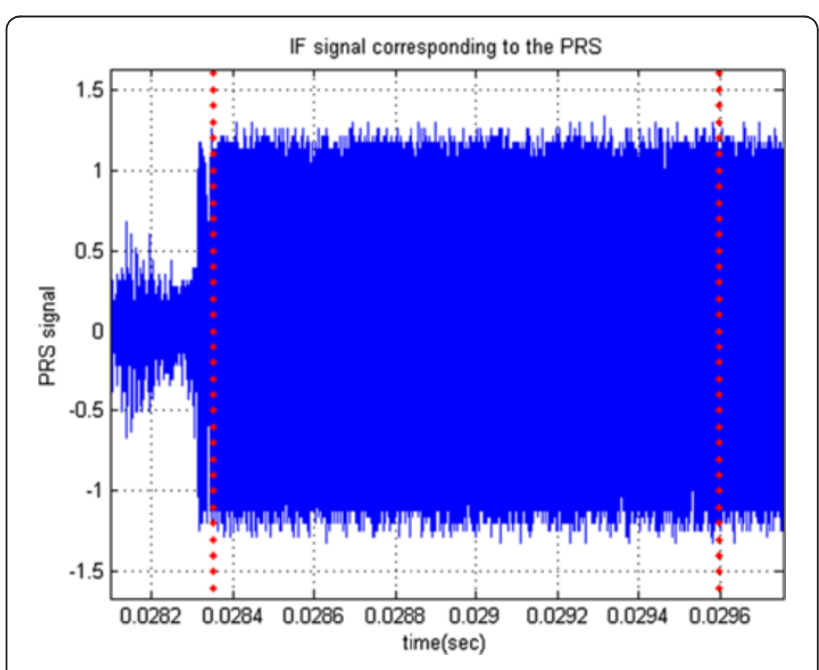

Figure 7 Actual IF signal for a phase reference symbol. 


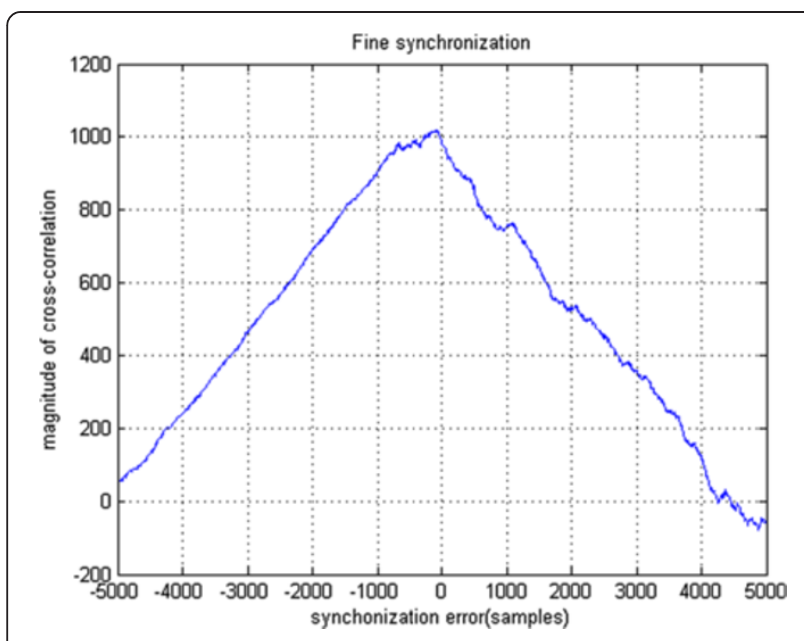

Figure 8 Result of the fine frame synchronization.

$\begin{aligned} P_{\text {receiver }}(t) & =\alpha_{\text {normal }} \times\left(P_{\text {first_arrived }}(t)+P_{\text {second_arrived }}(t)\right) \\ & =\alpha_{\text {normal }} \cdot P_{\text {first_arrived }}(t)+\alpha_{\text {normal }} \cdot \alpha_{\text {relative }} \cdot P_{\text {first_arrived }}\left(t-t_{\mathrm{d}}\right)\end{aligned}$

Here, $\alpha_{\text {normal }}$ is a scale factor that normalizes the magnitude of the received signal, and $t_{\mathrm{d}}$ is the delay time.

Using Equation 10, the autocorrelation of the ideal PRS signal at the IF stage can be calculated when the mobile user receives T-DMB signals from multiple transmitters.

$$
R(\delta)=E\left[P_{\text {receiver }}(t) \times P_{\text {receiver }}(t+\delta)\right]
$$

Here, $P_{\text {receiver }}(t)$ is the ideal received signal at the IF stage in the T-DMB receiver.

If Equation 10 is applied to Equation 11, Equation 12 can be derived.

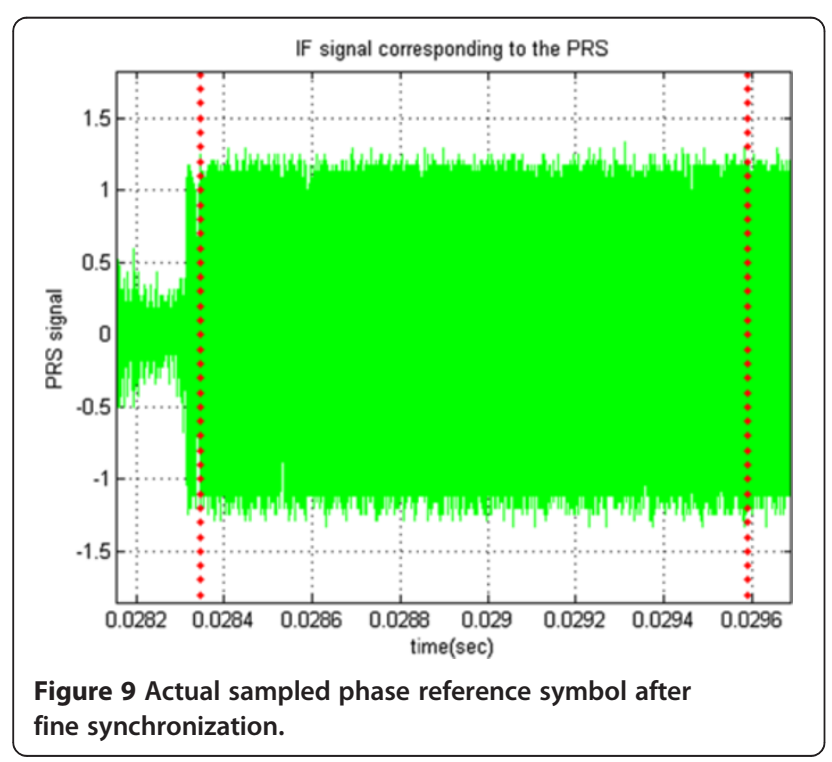

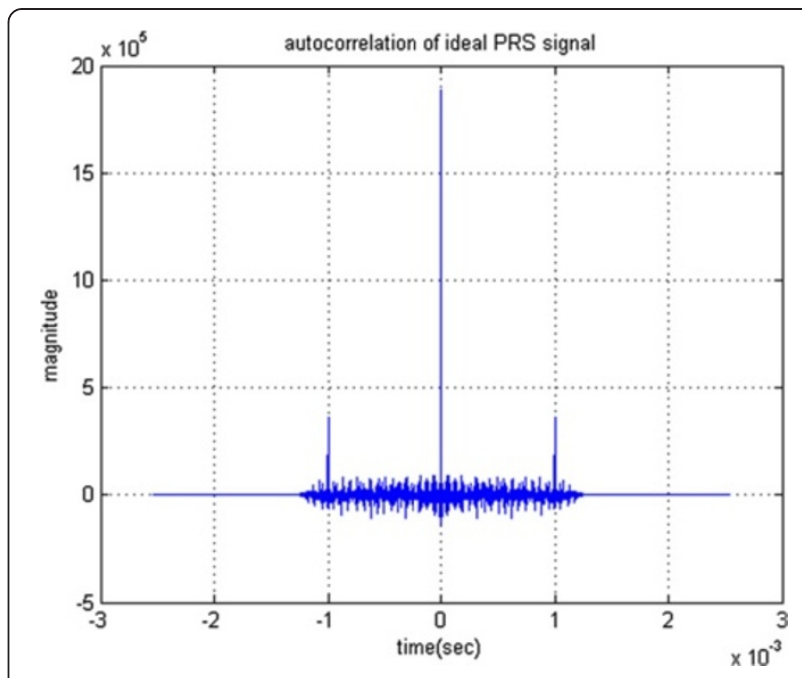

Figure 10 Autocorrelation of the ideal SC.

$$
\begin{aligned}
& R(\delta)=\left(\alpha_{\text {normal }}\right)^{2} E\left[P_{\text {first_arrived }}(t) P_{\text {first_arrived }}(t+\delta)\right] \\
& +\left(\alpha_{\text {normal }}\right)^{2} \alpha_{\text {relative }} E\left[P_{\text {first_arrived }}(t) P_{\text {first_arrived }}\left(t+\delta-t_{\mathrm{d}}\right)\right] \\
& +\left(\alpha_{\text {normal }}\right)^{2} \alpha_{\text {relative }} E\left[P_{\text {first_arrived }}\left(t+\delta-t_{\mathrm{d}}\right) P_{\text {first_arrived }}(t)\right] \\
& +\left(\begin{array}{lll}
\alpha_{\text {normal }} & \left.\alpha_{\text {relative }}\right)^{2} E\left[P_{\text {first_arrived }}(t+d) P_{\text {first_arrived }}\left(t+\delta-t_{\mathrm{d}}\right)\right]
\end{array}\right.
\end{aligned}
$$

Because the autocorrelation of the ideal PRS signal shows the impulse characteristic in the time domain, the expectation terms in Equation 12 can be replaced as follows in Equation 13:

$$
E\left[P_{\text {first_arrived }}(t) \times P_{\text {first_arrived }}(t+\delta)\right]=\delta(t)
$$

Here, $\delta(t)$ is a delta function.

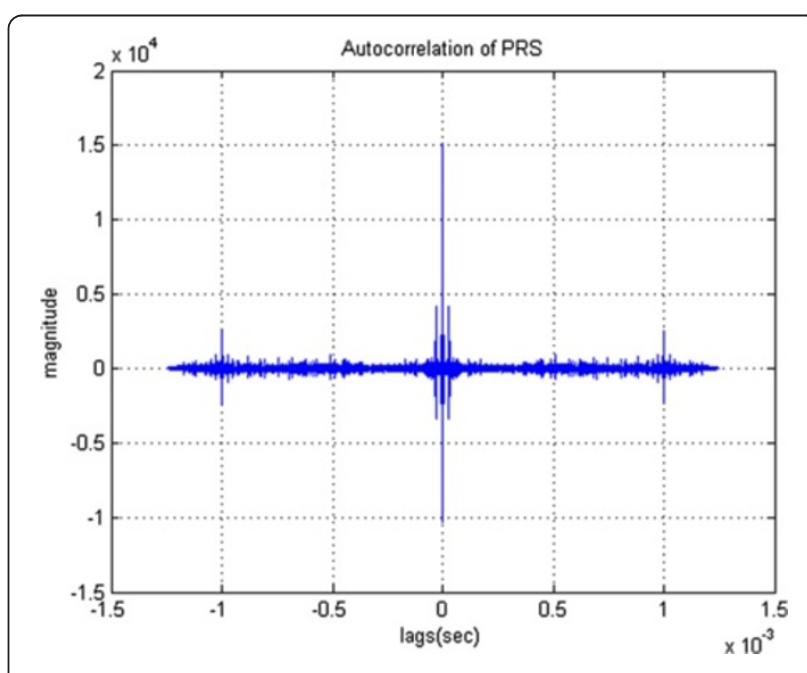

Figure 11 Autocorrelation of the actual received PRS. 


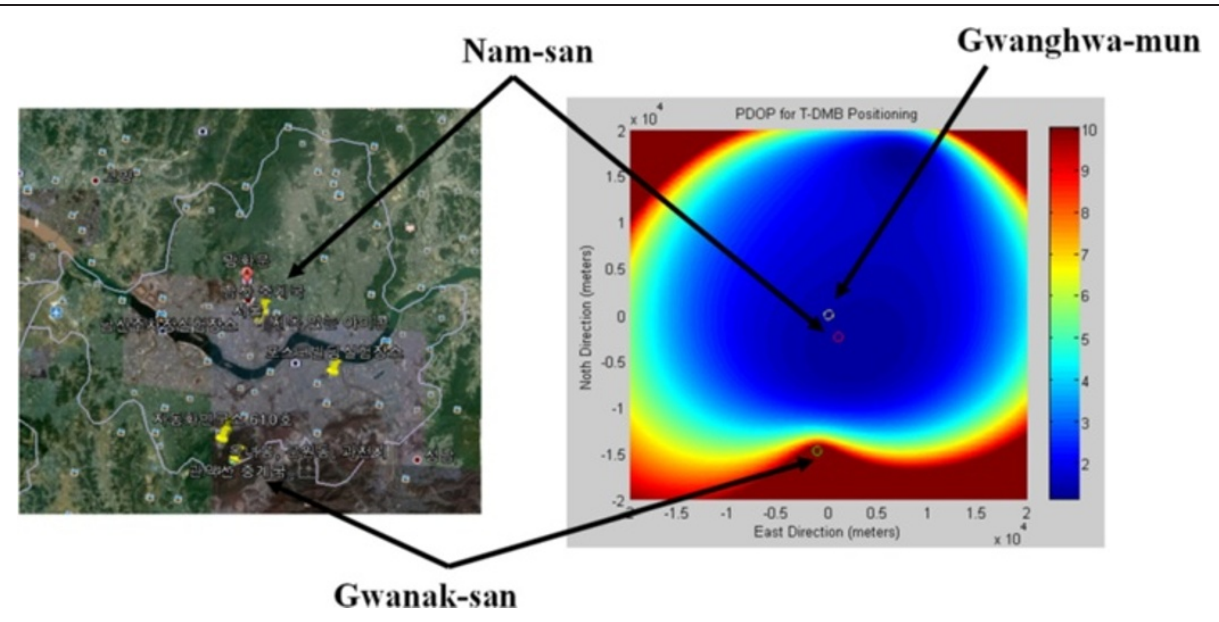

Figure 12 PDOP for Seoul.

Equation 12 can then be rewritten as follows:

$$
\begin{aligned}
R(t) & =\left(\alpha_{\text {normal }}\right)^{2} \times \delta(t) \\
& +\left(\alpha_{\text {normal }}\right)^{2} \times \alpha_{\text {relative }} \times \delta\left(t+t_{\mathrm{d}}\right) \\
& +\left(\alpha_{\text {normal }}\right)^{2} \times \alpha_{\text {relative }} \times \delta\left(t-t_{\mathrm{d}}\right) \\
& +\left(\alpha_{\text {normal }} \times \alpha_{\text {relative }}\right)^{2} \times \delta(t)
\end{aligned}
$$

Referring to Equation 14, a delayed signal with the time delay of $t_{\mathrm{d}}$ induces side peaks around the main peak at the center in the autocorrelation result, contrary to the case of the ideal PRS signal in Figure 10. In addition, the autocorrelation result of the actual sampled PRS signal can be considered as the sum of two components. One component is the sum of the autocorrelation results of the original PRS signal and the delayed signal, and the other is the crosscorrelation result of the original PRS signal and the delayed signal. Here, the received signal strength and the transmitter identification information (TII) for the received T-DMB signals can be used to determine the earliest signal to the mobile user.

\section{T-DMB positioning}

\section{TDOA positioning}

The T-DMB positioning system adopts the TDOA positioning technique to determine the mobile user position because the user receiver is not synchronized with the transmitter network, while the T-DMB transmitters are
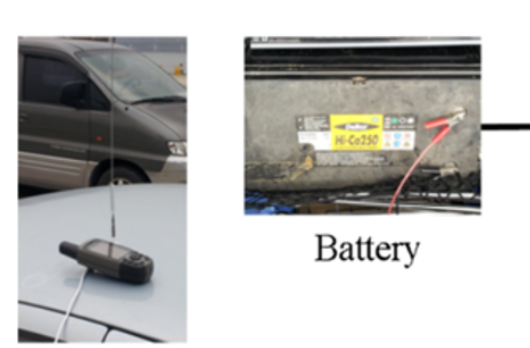

Battery

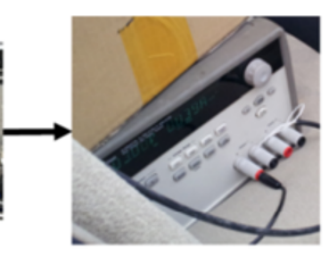

Power Supply

Antenna
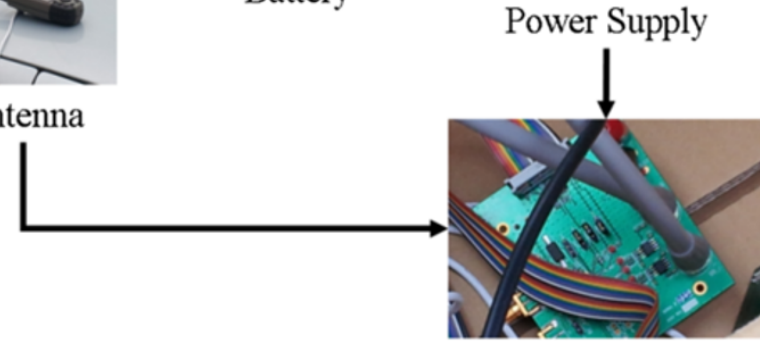

T-DMB RF receiver
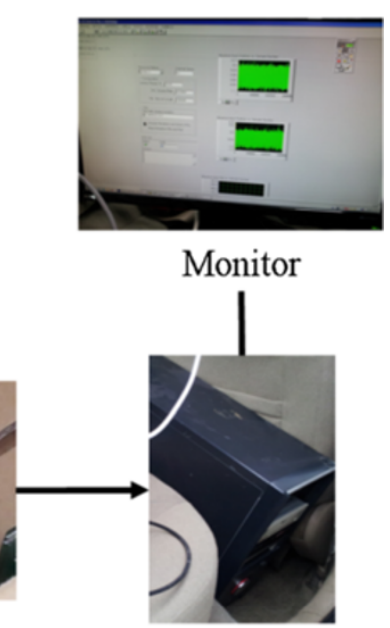

Computer

(NI-5112/

Receiver Control/

Signal Log)

Figure 13 Experimental set-up. 
Table 5 Position of experimental sites

\begin{tabular}{lccc}
\hline Site & Longitude (eastern, deg) & Latitude (northern, deg) & Height $(\mathbf{m})$ \\
\hline Seoul National University (outdoor, near Gwanak-san) & 126.95103 & 37.45014 & 186 \\
Han River-Ichon (outdoor, between Nam-san and Gwanak-san) & 126.97049 & 37.51762 & 37.55409 \\
Nam-san Park (outdoor, near Nam-san) & 126.98113 & 37.45435 & 13 \\
Laboratory (indoor, near Gwanak-san) & 126.95024 & 37.50553 & 127 \\
St. Tehran (urban) & 127.05561 & 46 \\
\hline
\end{tabular}

synchronized with each other. This means that the mobile user receiver can only measure the time difference of arrival from all audible T-DMB transmitters. The $i$-th and $j$-th transmitters are assumed to be at $\vec{R}_{i}=\left(x_{i}, y_{i}, z_{i}\right)$ and $\vec{R}_{j}=\left(x_{j}, y_{j}, z_{j}\right)$, and the mobile user is at $\vec{R}_{u}=\left(x_{u}, y_{u}, z_{u}\right)$. If there are two audible T-DMB transmitters to the user receiver, then the TDOA measurement for corresponding transmitters can be expressed using the following notations:

$T_{\mathrm{s}}$ is the system time (absolute time) at which the signal left the T-DMB transmitter.

$T_{\mathrm{u}}$ is the system time (absolute time) at which the signal reached the user receiver.

$\delta t$ is the offset of the T-DMB transmitter clock from the system time (advance is positive; retardation (delay) is negative).

$t_{\mathrm{u}}$ is the offset of the receiver clock from the system time.

$T_{\mathrm{s}}+\delta t$ is the T-DMB transmitter clock reading at the time that the signal left the T-DMB transmitter.

$T_{\mathrm{u}}+t_{\mathrm{u}}$ is the user receiver clock reading at the time when the signal reached the user receiver.

$c$ is the speed of light.

$\Delta t_{i}$ is the flight time from the $i$-th transmitter to the user receiver.
Considering offsets for both the transmitter and the user receiver, the TDOA for the $i$-th and $j$-th transmitters, which can be represented as $t_{i, j}$, can be measured as follows:

$$
\begin{aligned}
t_{i, j} & =\Delta t_{i}-\Delta t_{j} \\
& =\left[\left(T_{i, \mathrm{u}}+t_{\mathrm{u}}-T_{\mathrm{s}}-\delta t_{i}\right)-\left(T_{j, \mathrm{u}}+t_{\mathrm{u}}-T_{\mathrm{s}}-\delta t_{j}\right)\right] \\
& =T_{i, \mathrm{u}}-T_{j, \mathrm{u}}-\delta t_{i}+\delta t_{j}
\end{aligned}
$$

In Equation 15, $T_{i, \mathrm{u}}$ and $T_{j, \mathrm{u}}$ are the receiver clock readings for the signal from the $i$-th and $j$-th transmitters, respectively. Since the T-DMB transmitters are synchronized with each other in terms of the GPS clock, the clock offsets of the T-DMB transmitters in Equation 15 can be neglected.

$$
t_{i, j} \simeq T_{i, \mathrm{u}}-T_{j, \mathrm{u}}
$$

This TDOA measurement can then be converted to the difference of geometric ranges by multiplying the speed of light with both sides of Equation 16.

$$
c \times t_{i, j}=r_{i, \mathrm{u}}-r_{j, \mathrm{u}}
$$

Here, $r_{i, \mathrm{u}}$ and $r_{j, \mathrm{u}}$ are the geometric ranges from the $i$-th and $j$-th transmitters, respectively, to the mobile user. With the mobile user at $\vec{R}_{u}=\left(x_{\mathrm{u}}, y_{\mathrm{u}}, z_{\mathrm{u}}\right)$, the difference of
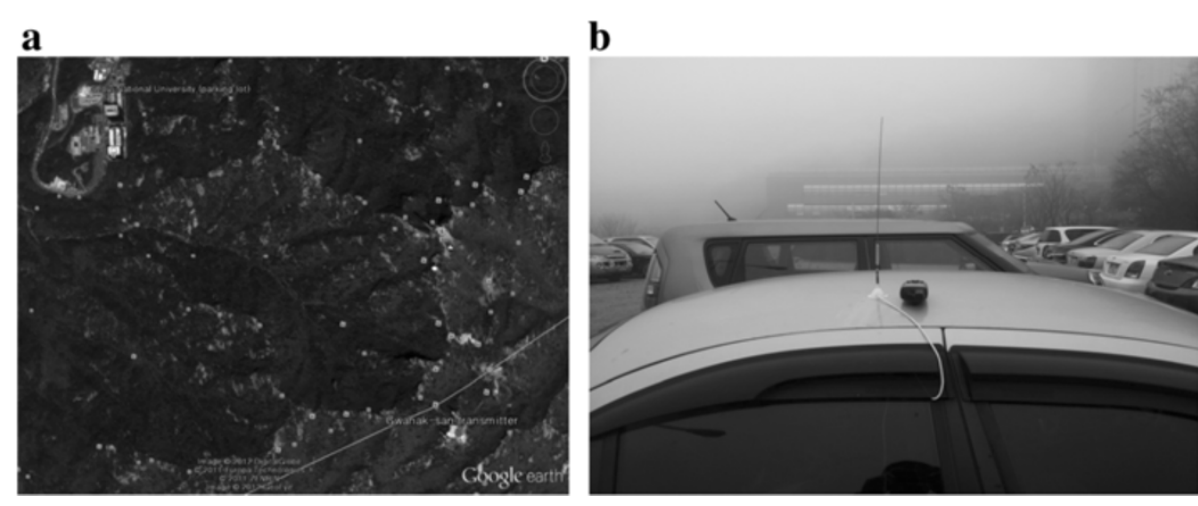

Figure 14 Seoul National University experimental site (a) and outdoor parking lot near building 301 in SNU (b). 

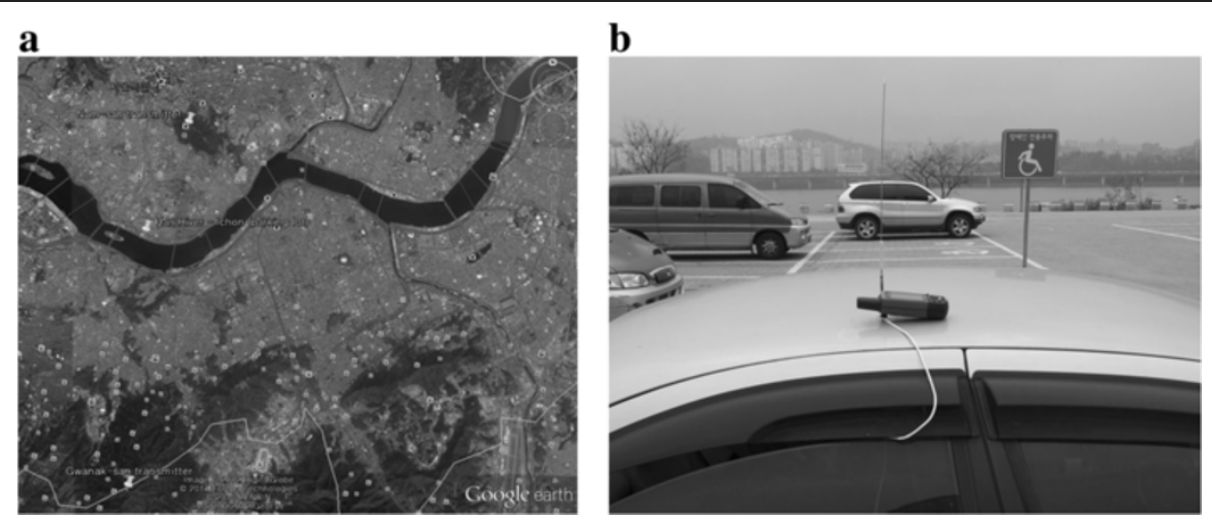

Figure 15 Han River-Ichon experimental site (a) and outdoor parking lot along Han River (b).

geometric ranges between the corresponding transmitters can be evaluated as a function of the mobile user position.

$$
\begin{aligned}
f\left(x_{\mathrm{u}}, y_{\mathrm{u}}, z_{\mathrm{u}}\right) & =r_{i, \mathrm{u}}-r_{j, \mathrm{u}} \\
& =\sqrt{\left(x_{i}-x_{\mathrm{u}}\right)^{2}+\left(y_{i}-y_{\mathrm{u}}\right)^{2}+\left(z_{i}-z_{\mathrm{u}}\right)^{2}} \\
& -\sqrt{\left(x_{j}-x_{\mathrm{u}}\right)^{2}+\left(y_{j}-y_{\mathrm{u}}\right)^{2}+\left(z_{j}-z_{\mathrm{u}}\right)^{2}}
\end{aligned}
$$

The initial estimate for the mobile user position in three dimensions $\hat{\vec{R}}_{\mathrm{u}}=\left(x_{\mathrm{u}}, y_{\mathrm{u}}, z_{\mathrm{u}}\right)$ can be used to evaluate an approximate of the TDOA measurement.

$$
\begin{aligned}
f\left(\hat{x}_{\mathrm{u}}, \hat{y}_{\mathrm{u}}, \hat{z}_{\mathrm{u}}\right) & =\hat{r}_{i, \mathrm{u}}-\hat{r}_{j, \mathrm{u}} \\
& =\sqrt{\left(x_{i}-\hat{x}_{\mathrm{u}}\right)^{2}+\left(y_{i}-\hat{y}_{\mathrm{u}}\right)^{2}+\left(z_{i}-\hat{z}_{\mathrm{u}}\right)^{2}} \\
& -\sqrt{\left(x_{j}-\hat{x}_{\mathrm{u}}\right)^{2}+\left(y_{j}-\hat{y}_{\mathrm{u}}\right)^{2}+\left(z_{j}-\hat{z}_{\mathrm{u}}\right)^{2}}
\end{aligned}
$$

The true mobile user position can then be considered as a sum of a current estimate and an estimate error as follows in Equation 20.

$$
\begin{aligned}
& x_{\mathrm{u}}=\hat{x}_{\mathrm{u}}+\delta x_{\mathrm{u}} \\
& y_{\mathrm{u}}=\hat{y}_{\mathrm{u}}+\delta y_{\mathrm{u}} \\
& z_{\mathrm{u}}=\hat{z}_{\mathrm{u}}+\delta z_{\mathrm{u}}
\end{aligned}
$$

Applying Equation 20 to Equation 18 leads to following equation:

$$
f\left(x_{\mathrm{u}}, y_{\mathrm{u}}, z_{\mathrm{u}}\right)=f\left(x_{\mathrm{u}}+\delta x_{\mathrm{u}}, y_{\mathrm{u}}+\delta y_{\mathrm{u}}, z_{\mathrm{u}}+\delta z_{\mathrm{u}}\right)
$$

If the current estimate is assumed to be close enough to the true position, then the Taylor series expansion can be applied to the right side of Equation 21.

$$
\begin{aligned}
& f\left(\hat{x}_{\mathrm{u}}+\Delta x_{\mathrm{u}}, \hat{y}_{\mathrm{u}}+\Delta y_{\mathrm{u}}, \hat{z}_{\mathrm{u}}+\Delta z_{\mathrm{u}}\right) \\
& \quad=f\left(\hat{x}_{\mathrm{u}}, \hat{y}_{\mathrm{u}}, \hat{z}_{\mathrm{u}}\right)+\frac{\partial f\left(\hat{x}_{\mathrm{u}}, \hat{y}_{\mathrm{u}}, \hat{z}_{\mathrm{u}}\right)}{\partial \hat{x}_{\mathrm{u}}} \times \Delta x_{\mathrm{u}}+\frac{\partial f\left(\hat{x}_{\mathrm{u}}, \hat{y}_{\mathrm{u}}, \hat{z}_{\mathrm{u}}\right)}{\partial \hat{y}_{\mathrm{u}}} \\
& \quad \times \Delta y_{\mathrm{u}}+\frac{\partial f\left(\hat{x}_{\mathrm{u}}, \hat{y}_{\mathrm{u}}, \hat{z}_{\mathrm{u}}\right)}{\partial \hat{z}_{\mathrm{u}}} \times \Delta z_{\mathrm{u}}+\text { H.O.T }
\end{aligned}
$$

Here, the partial derivatives over the second-order (higher order terms) are omitted to avoid non-linearity. Moreover, the first-order partial derivatives are evaluated as follows:
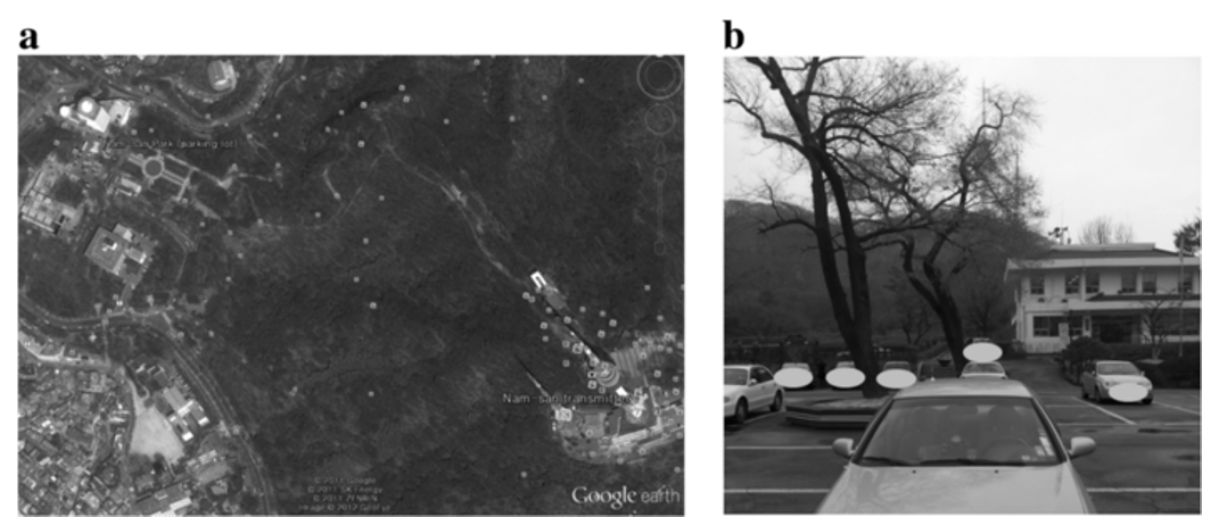

Figure 16 Nam-san Park experimental site (a) and outdoor parking lot near the Nam-san transmitter (b). 


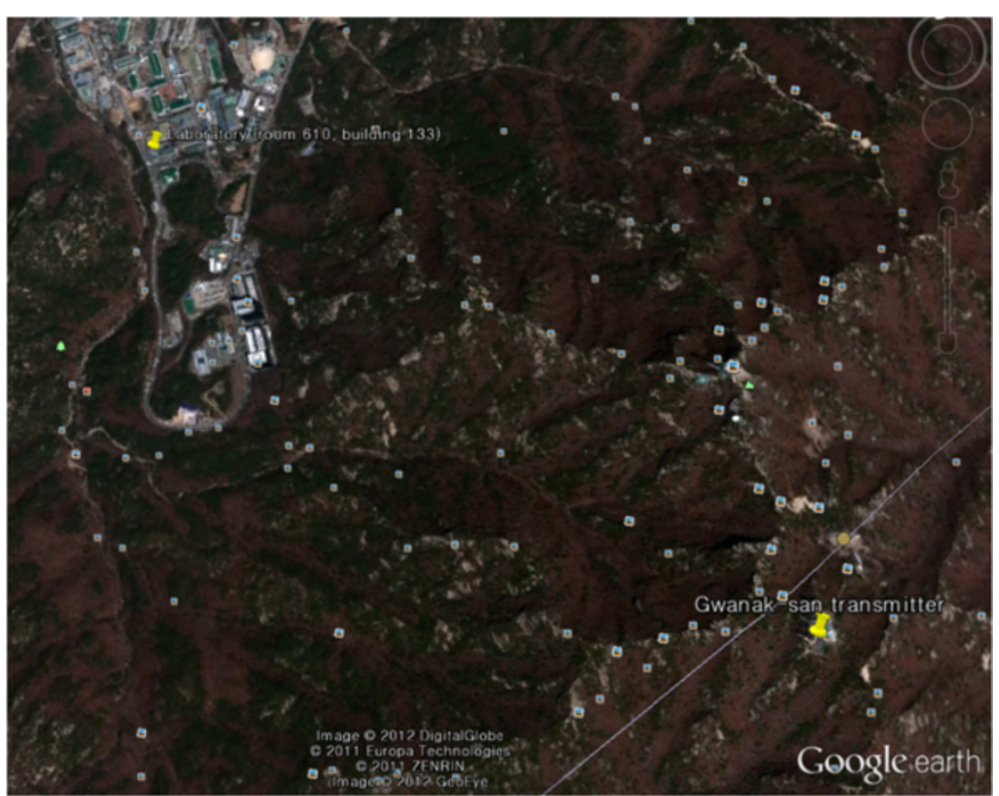

Figure 17 Laboratory experimental site (room 610, building 133).

$$
\begin{aligned}
& \frac{\partial f\left(\hat{x}_{\mathrm{u}}, \hat{y}_{\mathrm{u}}, \hat{z}_{\mathrm{u}}\right)}{\partial \hat{x}_{\mathrm{u}}}=-\frac{x_{i}-\hat{x}_{\mathrm{u}}}{\hat{r}_{i, \mathrm{u}}}+\frac{x_{j}-\hat{x}_{\mathrm{u}}}{\hat{r}_{j, \mathrm{u}}} \\
& \frac{\partial f\left(\hat{x}_{\mathrm{u}}, \hat{y}_{\mathrm{u}}, \hat{z}_{\mathrm{u}}\right)}{\partial \hat{y}_{\mathrm{u}}}=-\frac{y_{i}-\hat{y}_{\mathrm{u}}}{\hat{r}_{i, \mathrm{u}}}+\frac{y_{j}-\hat{y}_{\mathrm{u}}}{\hat{r}_{j, \mathrm{u}}} \\
& \frac{\partial f\left(\hat{x}_{\mathrm{u}}, \hat{y}_{\mathrm{u}}, \hat{z}_{\mathrm{u}}\right)}{\partial \hat{z}_{\mathrm{u}}}=-\frac{z_{i}-\hat{z}_{\mathrm{u}}}{\hat{r}_{i, \mathrm{u}}}+\frac{z_{j}-\hat{z}_{\mathrm{u}}}{\hat{r}_{j, \mathrm{u}}}
\end{aligned}
$$

Equation 21 can then be expanded as follows:

$$
\begin{aligned}
c \times t_{i, j}=c \times \hat{t}_{i, j} & +\left(-\frac{x_{i}-\hat{x}_{\mathrm{u}}}{\hat{r}_{i, \mathrm{u}}}+\frac{x_{j}-\hat{x}_{\mathrm{u}}}{\hat{r}_{j, \mathrm{u}}}\right) \times \delta x_{\mathrm{u}} \\
& +\left(-\frac{y_{i}-\hat{y}_{\mathrm{u}}}{\hat{r}_{i, \mathrm{u}}}+\frac{y_{j}-\hat{y}_{\mathrm{u}}}{\hat{r}_{j, \mathrm{u}}}\right) \times \delta y_{\mathrm{u}} \\
& +\left(-\frac{z_{i}-\hat{z}_{\mathrm{u}}}{\hat{r}_{i, \mathrm{u}}}+\frac{z_{j}-\hat{z}_{\mathrm{u}}}{\hat{r}_{j, \mathrm{u}}}\right) \times \delta z_{\mathrm{u}}
\end{aligned}
$$

Introducing a new variable, $\Delta t_{i, j}$, which represents the difference between the TDOA measurement and the calculated TDOA value using the current mobile user position estimate, induces the following Equation 25.

$$
\begin{aligned}
c \times \Delta t_{i, j} & =c\left(\hat{t}_{i, j}-t_{i, j}\right)=\left(\frac{x_{i}-\hat{x}_{\mathrm{u}}}{\hat{r}_{i, \mathrm{u}}}-\frac{x_{j}-\hat{x}_{\mathrm{u}}}{\hat{r}_{j, \mathrm{u}}}\right) \times \delta x_{\mathrm{u}} \\
& +\left(\frac{y_{i}-\hat{y}_{\mathrm{u}}}{\hat{r}_{i, \mathrm{u}}}-\frac{y_{j}-\hat{y}_{\mathrm{u}}}{\hat{r}_{j, \mathrm{u}}}\right) \times \delta y_{\mathrm{u}}+\left(\frac{z_{i}-\hat{z}_{\mathrm{u}}}{\hat{r}_{i, \mathrm{u}}}-\frac{z_{j}-\hat{z}_{\mathrm{u}}}{\hat{r}_{j, \mathrm{u}}}\right) \times \delta z_{\mathrm{u}}
\end{aligned}
$$

By integrating Equation 25 for four T-DMB transmitters that range from 1 to 4 , the following equation can be developed:

$$
\Delta \vec{z}=H \times \Delta \vec{p}
$$

Here,

$H=\left[\begin{array}{llll}\frac{x_{1}-\hat{x}_{\mathrm{u}}}{\hat{r}_{1}}-\frac{x_{2}-\hat{x}_{\mathrm{u}}}{\hat{r}_{2}} & \frac{y_{1}-\hat{y}_{\mathrm{u}}}{\hat{r}_{1}}-\frac{y_{2}-\hat{y}_{\mathrm{u}}}{\hat{r}_{2}} & \frac{z_{1}-\hat{z}_{\mathrm{u}}}{\hat{r}_{1}}-\frac{z_{2}-\hat{z}_{\mathrm{u}}}{\hat{r}_{2}} \\ \frac{x_{1}-\hat{x}_{\mathrm{u}}}{\hat{r}_{1}}-\frac{x_{3}-\hat{x}_{\mathrm{u}}}{\hat{r}_{3}} & \frac{y_{1}-\hat{y}_{\mathrm{u}}}{\hat{r}_{1}}-\frac{y_{3}-\hat{y}_{\mathrm{u}}}{\hat{r}_{3}} & \frac{z_{1}-\hat{z}_{\mathrm{u}}}{\hat{r}_{1}}-\frac{z_{3}-\hat{z}_{\mathrm{u}}}{\hat{r}_{3}} \\ \frac{x_{1}-\hat{x}_{\mathrm{u}}}{\hat{r}_{1}}-\frac{x_{4}-\hat{x}_{\mathrm{u}}}{\hat{r}_{4}} & \frac{y_{1}-\hat{y}_{\mathrm{u}}}{\hat{r}_{1}}-\frac{y_{4}-\hat{y}_{\mathrm{u}}}{\hat{r}_{4}} & \frac{z_{1}-\hat{z}_{\mathrm{u}}}{\hat{r}_{1}}-\frac{z_{4}-\hat{z}_{\mathrm{u}}}{\hat{r}_{4}}\end{array}\right]$,

$\Delta \vec{p}=\left[\begin{array}{l}\Delta x_{\mathrm{u}} \\ \Delta y_{\mathrm{u}} \\ \Delta z_{\mathrm{u}}\end{array}\right], \Delta \vec{z}=\left[\begin{array}{l}c \times \Delta t_{1,2} \\ c \times \Delta t_{1,3} \\ c \times \Delta t_{1,4}\end{array}\right]$

The measurement matrix $\Delta \vec{z}$ contains the difference in information between TDOA measurements and estimates, while the geometric matrix $H$ contains the differences of directional cosine vectors from the mobile user to the corresponding T-DMB transmitters. The correction matrix $\Delta \vec{p}$ is a collection of estimate errors for the mobile user position. The solution of Equation 26 is known to be as follows [30-33].

$$
\Delta \vec{p}=\left(H^{T} \times H\right)^{-1} \times H^{T} \times \Delta \vec{z}
$$

With iterative updates of the current estimate with the correction matrix, the final estimate can be solved to determine the mobile user position. To expect the positioning error of the proposed T-DMB positioning method, a magnitude of $\left(H^{T} \times H\right)^{-1}$, which is a geometric dilution of precision (DOP), is investigated for the capital area of Korea as shown in Figure 12 [34,35]. Most of the area shows the DOP of about 2. This means that the position error for the T-DMB positioning can be 


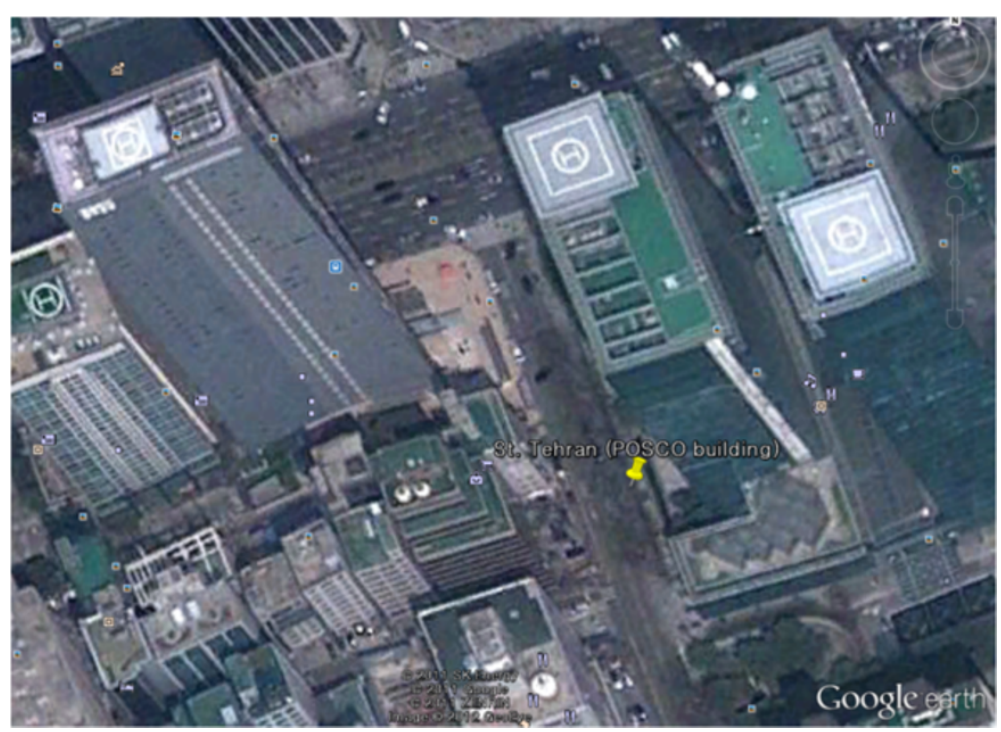

Figure 18 St. Tehran experimental site (near the POSCO building). The root mean square (RMS) error for TDOA measurements at all experimental sites is as follows.

expected to be about double $\sigma_{\text {UERE}}$, which is a user equivalent range error for the T-DMB positioning system.

\section{Experimental results and analysis \\ Experimental results on TDOA measurements}

Experiments and analysis are performed to certify the possibility of the proposed positioning method. For experiments, the MAX2170 EVKIT from the MAXIM semiconductor cooperation and the PCI-5112 data acquisition board (DAQ) from the National Instruments cooperation are used to capture the intermediate frequency (IF) signal in forms of in-phase and out-phase (I/Q) from the received T-DMB signal. Moreover, the position of the experimental sites and T-DMB transmitters are measured using GPS map 60CS from the GARMIN.

The MAX2170 EVKIT is the evaluation board of the MAX2170 T-DMB RF tuner chip, which receives the transmitted T-DMB signal and demodulates these signals to the output I/Q signal at the baseband frequency [36]. The $I / Q$ signal is then sampled at a frequency that is sufficiently higher than the baseband frequency, 2.048 megahertz (MHz), using the DAQ, PCI-5112 [37].

Also, the sampled signals are post-processed using MATLAB to certify the feasibility of the proposed positioning method. Figure 13 shows the experimental set-up.

The proposed method is used to measure the TDOA measurements at several sites in Seoul, in order to verify the performance of the proposed positioning method. Table 5 shows the position of the experimental sites. The position information is measured by GPS map 60CS
(GARMIN). The mean of the measurements for $100 \mathrm{~s}$ is assumed to be the true position. The specification states that $95 \%$ of the measurements are guaranteed to have a positioning error of less than $15 \mathrm{~m}$ [38].

In Table 5, for the Laboratory site and the St. Tehran site, the Google map is used to estimate GPS position, because the GPS position determination is not accurate in indoor and urban environments. The experimental site for the Seoul National University is shown in Figure 14a. It is an outdoor parking lot, which is near building 301, as shown in Figure 14b.

The experiment for Ichon is also conducted at a parking lot, which is located between the Nam-san transmitter and the Gwanak-san transmitter (Figure 15). This site is expected to have LOS from both the Gwanak-san transmitter and the Nam-san transmitter.

Figure 16a shows the experimental site for the Nam-san Park, which is near the Nam-san transmitter and is guaranteed to have LOS from the T-DMB transmitter to the user receiver. In Figure 16b, the Nam-san T-DMB transmitter can be seen beyond the experiment vehicle in the bottom of the figure.

\section{Table 6 Experimental results}

\begin{tabular}{lccc}
\hline Site & $\begin{array}{c}\text { True TDOA } \\
(\mathbf{m})\end{array}$ & \multicolumn{2}{c}{ Measurement error (RMS) } \\
\cline { 3 - 4 } & & $\mathbf{( m )}$ & $\mathbf{( \% )}$ \\
\hline SNU (parking lot) & $10,042.333$ & 256.973 & -7.224 \\
Han River-Ichon & $4,573.519$ & 49.293 & -2.821 \\
Nam-san Park & $11,990.700$ & 118.890 & 3.117 \\
Laboratory & $9,208.700$ & 96.239 & -1.723 \\
St. Tehran & $3,064.984$ & 26.103 & 0.963 \\
\hline
\end{tabular}




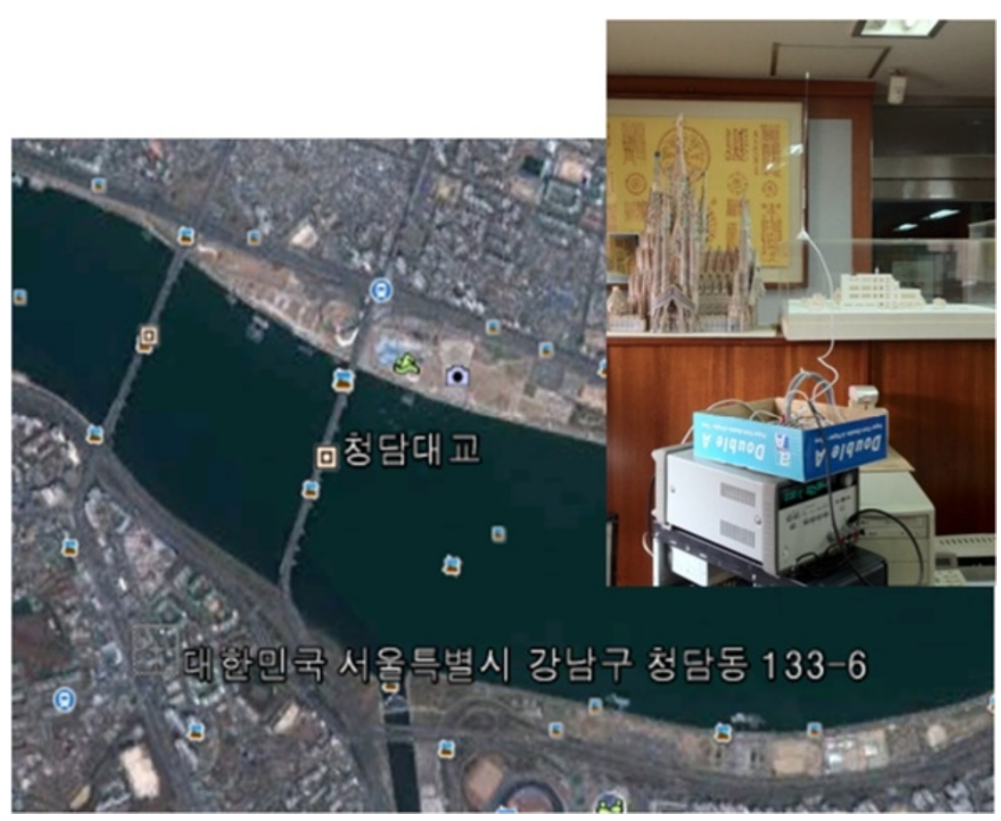

Figure 19 Office in Cheongdam-dong. With more than 50 experiments at the same place, the error distribution results are as follows.

Figure 17 shows the experimental site for an indoor environment. It is room 610, building 133, Seoul National University.

In addition, an experiment for an urban environment was also performed in St. Tehran (Figure 18); where, because of the high-rise buildings, GPS positioning error is known to increase.

The root mean square (RMS) error for TDOA measurements at all experimental sites is shown in Table 6.

To check the error distribution of the TDOA measurements, more experiments were performed in St. Tehran and at an office in Cheongdam-dong. The previous experiment was also conducted at St. Tehran, and the office is on the 3rd floor, 133-6, Cheongdam-dong, Gangnam-gu, Seoul, Korea (Figure 19).

With more than 50 experiments at the same place, the error distribution results are shown in Table 7.

\section{Analysis on TDOA measurement errors}

From the experimental results, the RMS errors for the TDOA measurements are less than $300 \mathrm{~m}$ for all sites in Seoul. For the sites where a T-DMB transmitter is close, the errors are larger than other cases. Since the T-DMB transmitters are located on the top of mountains, the

Table 7 Standard deviation of TDOA measurement error

\begin{tabular}{lc}
\hline Experimental site & Standard deviation of error $(\mathbf{m})$ \\
\hline St. Tehran & 28.357 \\
Cheongdam-dong (indoor) & 30.3184 \\
Cheongdam-dong (outdoor) & 39.5476 \\
\hline
\end{tabular}

diffraction may affect the measurements. The measurement errors are small for both St. Tehran and Ichon. This demonstrates that the outer walls of the building are not the main barrier for the T-DMB positioning. The standard deviation of the TDOA measurement errors is about $30 \mathrm{~m}$. Although this error characteristic is worse than that of the GPS pseudo-range, it is sufficient for use in the positioning system. Moreover, the accuracy and the error characteristic do not decrease, even in indoor and urban environments. This could be the strength of the T-DMB positioning method. Finally, for the area where the DOP is 2, the error for the mobile user position determination is expected to be smaller than $600 \mathrm{~m}$ in all environments: outdoor, indoor, and urban. Especially, if the mobile user is not close to the T-DMB transmitter, then the positioning error could be bounded to less than $200 \mathrm{~m}$, even in indoor and urban environments.

\section{Conclusion}

In this paper, a new positioning method which utilizes the T-DMB signal is presented to serve the seamless position determination to the mobile user both in indoor and outdoor environments. The proposed method uses only a single T-DMB receiver to measure the TDOA between multiple audible T-DMB transmitter pairs. In addition, it does not need any extra infrastructure for the position determination because it only uses the PRS in the T-DMB signal.

Experiments for measuring the TDOA using the actual $\mathrm{T}-\mathrm{DMB}$ receiver at several sites in Seoul have been 
performed to certify that the proposed method is feasible in both indoor and outdoor environments. From the results, the accuracy of TDOA measurements is bounded to less than $300 \mathrm{~m}$ even in indoor and urban environments. Moreover, the variance of the error distribution is about $30 \mathrm{~m}$, which is small enough for use in the positioning system. However, the position determination of the mobile user with the actual T-DMB signal could not be performed because only two T-DMB transmitters are audible in most areas of Seoul. With enough T-DMB transmitters in the future, the mobile-user position could be determined by using the T-DMB transmission signal alone.

In addition, the T-DMB positioning method presented in this paper provides the opportunity to determine the mobile user position in challenging environments. Therefore, it can be demonstrated that the proposed method opens a new possibility for seamless positioning.

\section{Competing interests}

The authors declare that they have no competing interests.

\section{Acknowledgement}

This work was supported by a grant from the Korea Research Council of Fundamental Science \& Technology funded by the Ministry of Education, Science and Technology in 2014 [Project: A Study on Satellite based Position Tracking Technology for Calamity Prevention and Public Safety Improvement]

\section{Author details}

'Department of Electrical Engineering, Konkuk University, Hwayang-dong, Gwangjin-gu, Seoul 143-701, South Korea. ${ }^{2}$ Division of Satellite Navigation, Space Application and Technology Centre, Korea Aerospace Research Institute (KARI), Yuseong, Daejeon 305-333, South Korea.

Received: 30 May 2013 Accepted: 5 June 2014

Published: 3 July 2014

\section{References}

1. B Rao, L Minakakis, Evolution of mobile location-based services. Commun. ACM 46(12), 61-65 (2003)

2. P Prasithsangaree, $P$ Krishnamurthy, PK Chrysanthis, On indoor position location with wireless LANs. The 13th IEEE International Symposium on Personal, Indoor and Mobile Radio. Communications 2, 720-724 (2002)

3. L Hui, D Houshang, B Pat, L Jing, Survey of wireless indoor positioning techniques and system. IEEE Trans. Syst. Man. Cybern. 37(6), 1067-1080 (2007)

4. L-W Chan, J-r Chiang, Y-c Chen, C-n Ke, J Hsu, H-h Chu, Collaborative localization enhancing WiFi-based position estimation with neighborhood links in clusters, PERVASIVE 2006. LNCS 3968, 50-66 (2006)

5. K Yu, I Oppermann, Performance of UWB position estimation based on time-of-arrival measurements, in Proceedings of the IEEE Conference Ultra Wideband Systems and Technologies (UWBST), Kyoto, Japan, 2004), pp. 400-404

6. LM Ni, Y Liu, YC Lau, AP Patil, LANDMARC: indoor location sensing using active RFID. Wireless Netw 10(6), 701-710 (2004)

7. BP Nissanka, C Anit, B Hari, The Cricket location-support system, in Proceedings of the 6th International Conference on Mobile Computing and Networking, Boston, MA, USA, 2000), pp. 32-43

8. H Martin, M Rudolf, S Markus, Estimating position and velocity of mobiles in a cellular radio network. IEEE Trans. Veh. Technol. 46(1), 65-71 (1997)

9. JJ Caffery, GL Stuber, Overview of radiolocation in CDMA cellular system. IEEE Commun. Mag. 36(4), 38-45 (1998)

10. C Drane, M Macnaughtan, C Scott, Positioning GSM telephones. IEEE Commun. Mag. 36(4), 46-54 (1998)

11. K Pahlavan, $X$ Li, J Makela, Indoor geolocation science and technology. IEEE Commun. Mag. 40(2), 112-118 (2002)
12. M Rabinowitz, JJ Spilker Jr, A new positioning system using television synchronization signals. IEEE Trans. Broadcast. 51(1), 51-61 (2005)

13. R Corporation, In-Building Timing and Location for Femtocells (Rosum FemtoSynch Technical Report, Mountain View, CA, USA, 2008)

14. L Pei, R Chen, Y Chen, H Leppäkoski, A Perttula, Indoor/outdoor seamless positioning technologies integrated on smart phone, in The First International Conference on Advances in Satellite and Space Communications, 2009, Colmar, France, 2009), pp. 141-145

15. X Liu, Q Man, L Henghui, X Lin, Wi-Fi/MARG/GPS integrated system for seamless mobile positioning, in IEEE Wireless Communications and Networking Conference (WCNC), Shanghai, 2013)

16. $E$ Jinlong, $M$ Jie, A research on seamless indoor and outdoor positioning J. Comput. 8(12), 2013 (2013)

17. L Gwangsoon, C Sammo, Y Kyu-Tae, KH Young, IL Soo, Development of terrestrial DMB transmission system based on Eureka-147 DAB system. IEEE Trans. Consum. Electron. 51(1), 63-68 (2005)

18. S Cho, GS Lee, B Bae, KT Yang, C-H Ahn, S-I Lee, C Ahn, System and services of terrestrial digital multimedia broadcasting (T-DMB). IEEE Trans. Broadcast. 53(1), 171-178 (2007)

19. The European Telecommunications Standards Institute, ETSI EN 300401 V1.4.1, Radio Broadcasting Systems, Digital audio broadcasting (DAB) to mobile, portable and fixed receivers (Sophia-Antipolis Cedex, France, 2006)

20. The European Telecommunications Standards Institute, ETSI TR 101 496-3 V1.1.2, Digital Audio Broadcasting (DAB); Guidelines and rules for implementation and operation, Part 3: Broadcasting network (Sophia-Antipolis Cedex, France, 2001)

21. Korea Communications Commission, Telecommunications Business Act. The Broadcasting Act no. 10166 (Korea Communications Commission, Seoul, 2010)

22. The European Telecommunications Standards Institute, ETSI TS 102 427, Digital Audio Broadcasting (DAB); Data Broadcasting - MPEG 2 TS Streaming (Sophia-Antipolis Cedex, France, 2005)

23. The European Telecommunications Standards Institute, ETSI TR 101 496-1, Digital Audio Broadcasting (DAB); Guidelines and rules for implementation and operation, Part 1: System outline (Sophia-Antipolis Cedex, France, 2000)

24. The European Telecommunications Standards Institute, ETS 300 799, Digital Audio Broadcasting (DAB); Distribution interfaces; Ensemble Transport Interface (ETI) (Sophia-Antipolis Cedex, France, 1998)

25. KH Kim, YK Ham, YH Kim, The terrestrial digital multimedia broadcasting system technology. Telecommunications Review 13, Seoul, South Korea, 2003), pp. 287-310

26. F Gerard, Single frequency networks: a magic feature of the COFDM (ST Engineering, Singapore, 1999), p. 9

27. W Hoeg, T Lauterbach, Digital Audio Broadcasting: Principles and Applications of $D A B, D A B+$ and $D M B$ 3/e (Wiley, New York, 2009)

28. $\mathrm{R}$ Van Nee, R Prasad, OFDM for Wireless Multimedia Communications (Artech House, Boston, USA, 2000)

29. Y Mostofi, Timing Synchronization and ICI Mitigation for Pilot-Aided OFDM Mobile Systems (Stanford University, 2003). Dissertation

30. B Fang, Simple solution for hyperbolic and related position fixes. IEEE Trans. Aerosp. Electron. Syst. 26(5), 748-753 (1990)

31. S Dong-Ho, S Tae-Kyung, Comparisons of error characteristics between TOA and TDOA positioning. IEEE Aerosp. Electron. Syst. 38(1), (2002)

32. WH Foy, Position-location solutions by Taylor-series estimation. IEEE Trans. Aerosp. Electron. Syst. AES-12, 187-194 (1976)

33. GB Moon, An Integrated T-DMB/GPS Navigation for Seamless Positioning in Outdoor and Indoor Environments (Electrical Engineering, Seoul National University, 2012). Dissertation

34. P Misra, P Enge, Global Positioning System-Signals, Measurements, and Performance (Ganga-Jamuna Press, Lincoln, 2004)

35. P Massatt, K Rudnick, Geometric formulas for dilution of precision calculations. Navigation 37(4), (1990-1991)

36. Maxim Integrated Products, MAX2170/MAX2171 Datasheet, 19-0699; Rev 3; 8/09 (San Jose, CA, USA).

37. National Instruments Corporation, NI PCI/PXI-5112 Specifications, 323495A-01 (Austin, Texas, USA, 2003)

38. Garmin, Garmin CS60 User Manua (Wichita, Kansas, USA, 2004)

\section{doi:10.1186/1687-6180-2014-99}

Cite this article as: Moon et al: A T-DMB navigation system for seamless positioning in both indoor and outdoor environments. EURASIP Journal on Advances in Signal Processing 2014 2014:99. 